\title{
Multi-omics analysis delineates the distinct functions of sub-cellular acetyl-CoA pools in Toxoplasma gondii
}

Joachim Kloehn ${ }^{1 \dagger}$, Rebecca D. Oppenheim ${ }^{1 \dagger}$, Ghizal Siddiqui ${ }^{2}$, Pieter-Jan De Bock ${ }^{3}$, Sunil Kumar Dogga', Yohann Coute ${ }^{3}$, Mohamed-Ali Hakimi ${ }^{4}$, Darren J. Creek ${ }^{2}$ and Dominique Soldati-Favre ${ }^{1 *}$

\begin{abstract}
Background: Acetyl-CoA is a key molecule in all organisms, implicated in several metabolic pathways as well as in transcriptional regulation and post-translational modification. The human pathogen Toxoplasma gondii possesses at least four enzymes which generate acetyl-CoA in the nucleo-cytosol (acetyl-CoA synthetase (ACS); ATP citrate lyase $(A C L)$ ), mitochondrion (branched-chain a-keto acid dehydrogenase-complex $(B C K D H))$ and apicoplast (pyruvate dehydrogenase complex (PDH)). Given the diverse functions of acetyl-CoA, we know very little about the role of sub-cellular acetyl-CoA pools in parasite physiology.

Results: To assess the importance and functions of sub-cellular acetyl-CoA-pools, we measured the acetylome, transcriptome, proteome and metabolome of parasites lacking ACL/ACS or BCKDH. We demonstrate that ACL/ACS constitute a synthetic lethal pair. Loss of both enzymes causes a halt in fatty acid elongation, hypo-acetylation of nucleo-cytosolic and secretory proteins and broad changes in gene expression. In contrast, loss of BCKDH results in an altered TCA cycle, hypo-acetylation of mitochondrial proteins and few specific changes in gene expression. We provide evidence that changes in the acetylome, transcriptome and proteome of cells lacking BCKDH enable the metabolic adaptations and thus the survival of these parasites.
\end{abstract}

Conclusions: Using multi-omics and molecular tools, we obtain a global and integrative picture of the role of distinct acetyl-CoA pools in T. gondii physiology. Cytosolic acetyl-CoA is essential and is required for the synthesis of parasite-specific fatty acids. In contrast, loss of mitochondrial acetyl-CoA can be compensated for through metabolic adaptations implemented at the transcriptional, translational and post-translational level.

Keywords: Toxoplasma gondii, Acetyl-CoA, Branched-chain a-keto acid dehydrogenase-complex (BCKDH), ATP citrate lyase (ACL), Acetyl-CoA synthetase (ACS), Acetylome, Multi-omics, Metabolism, Phosphoenolpyruvate carboxykinase (PEPCK), Formate/nitrite transporter (FNT)

\footnotetext{
* Correspondence: Dominique.Soldati-Favre@unige.ch

† Joachim Kloehn and Rebecca D. Oppenheim contributed equally to this work.

'Department of Microbiology and Molecular Medicine, CMU, University of Geneva, Rue Michel-Servet 1, 1211 Geneva, Switzerland

Full list of author information is available at the end of the article
}

(c) The Author(s). 2020 Open Access This article is licensed under a Creative Commons Attribution 4.0 International License, which permits use, sharing, adaptation, distribution and reproduction in any medium or format, as long as you give appropriate credit to the original author(s) and the source, provide a link to the Creative Commons licence, and indicate if changes were made. The images or other third party material in this article are included in the article's Creative Commons licence, unless indicated otherwise in a credit line to the material. If material is not included in the article's Creative Commons licence and your intended use is not permitted by statutory regulation or exceeds the permitted use, you will need to obtain permission directly from the copyright holder. To view a copy of this licence, visit http://creativecommons.org/licenses/by/4.0/ The Creative Commons Public Domain Dedication waiver (http://creativecommons.org/publicdomain/zero/1.0/) applies to the data made available in this article, unless otherwise stated in a credit line to the data. 


\section{Background}

The phylum Apicomplexa groups a range of obligate intracellular parasites including Plasmodium spp., Cryptosporidium parvum and Toxoplasma gondii, the causative agents of malaria, gastrointestinal disease and toxoplasmosis, respectively. The metabolism of these pathogens is an area of intense research, aiming to identify new drug targets and develop inhibitors with new chemotypes to overcome the limitations of current drugs, such as high cost, toxicity and emerging resistance $[1,2]$.

T. gondii harbours several metabolically active subcellular compartments including the cytosol, nucleus, endoplasmic reticulum (ER), Golgi apparatus, mitochondrion and apicoplast, a relict plastid-like organelle derived from secondary endosymbiosis and possibly peroxisomes, which may form in the oocyst/sporozoite stage [3-5]. Acetyl-CoA is a hub metabolite with distinct and crucial functions in each of these compartments, involved in several anabolic and catabolic pathways and crucial for the acetylation of histones as well as non-histone proteins [69]. Due to its amphiphilic nature and high molecular weight, acetyl-CoA cannot freely cross the membranes and must be produced within, or actively transported into, the compartments which rely on acetyl-CoA (Fig. 1a) [9]. In $T$. gondii, the pyruvate dehydrogenase complex (PDH) converts pyruvate to acetyl-CoA in the apicoplast [12]. In the mitochondrion, the branched-chain $\alpha$-keto acid dehydrogenase-complex (BCKDH) replaces the function of PDH to generate acetyl-CoA from pyruvate [13]. Two complementary routes generate acetyl-CoA in the cytosol and nucleus: the acetyl-CoA synthetase (ACS) produces acetyl-CoA from acetate, and the ATP citrate lyase (ACL) converts citrate to acetyl-CoA [14]. A putative acetyl-CoA transporter (AT-1) likely enables the import of cytosolic acetyl-CoA into the ER [14, 15]. If, and during which life cycle stages, acetyl-CoA is generated by $\beta$-oxidation in $T$. gondii is unclear [5].

The high negative scores in a recent fitness screen of $T$. gondii metabolic genes indicate fitness-conferring roles of PDH and BCKDH (Fig. 1b) [16]. In contrast, the low positive scores of ACS and ACL indicate dispensability, consistent with a previous study which demonstrated that ACS and ACL are synthetic lethal [14]. Apart from coccidians, other apicomplexans, including the malaria parasites, lack ACL and thus rely solely on ACS to generate acetyl-CoA in the nucleo-cytosol (Fig. 1b). Consequently, ACS is predicted to be essential in Plasmodium as indicated by genome-wide fitness screens and is considered as a promising drug target $[10,17,18]$.

While previous studies focused on determining the extent of protein acetylation in apicomplexans and identified differences between parasite stages or strains [19-22], little is known about the specific roles of distinct acetyl-CoA pools and how these impact parasite physiology. Here, we combined multi-omics analysis with molecular tools to reveal the diverse functions of acetyl-CoA in T. gondii.

\section{Results}

Loss of nucleo-cytosolic acetyl-CoA production is detrimental and causes morphological defects in $T$. gondii We have previously postulated that both ACL and ACS contribute to the generation of acetyl-CoA in the cytosol and nucleus and constitute a synthetic lethal pair in $T$. gondii [14]. To elucidate the role of ACL and ACS, we generated an inducible conditional knock-down of ACS in $\mathrm{RH}$ parasites (i $\triangle \mathrm{ACS}$ ) as well as in parasites in which acl was deleted by double-homologous recombination $(\triangle \mathrm{ACL} / \mathrm{i} \triangle \mathrm{ACS})$. For the conditional knock-down, a destabilisation domain (DD) was fused to a myc-tag at the $\mathrm{N}$-terminus of ACS in the endogenous acs gene locus by CRISPR/Cas9-mediated genome editing (Additional file 1: Figure S1a). The DD allows for rapid proteasome degradation in the absence of the protective ligand Shield-1 (Shld-1) [23]. Integration of the DDmyc in the acs locus and replacement of the acl open reading frame (ORF) with the hypoxanthine-xanthine-guanine phosphoribosyl transferase (HXGPRT) resistance cassette (Additional file 1: Figure S1a, b) were validated by PCR analysis of genomic DNA (Additional file 1: Figure S1c). Expression and effective regulation of the DDmyc-ACS fusion protein in $\mathrm{i} \triangle \mathrm{ACS}$ and $\triangle \mathrm{ACL} / \mathrm{i} \triangle \mathrm{ACS}$ parasites were confirmed by western blot (Fig. 1c).

In plaque assays, which assess several lytic cycles, i $\triangle \mathrm{ACS}$ or $\triangle \mathrm{ACL}$ parasites showed no defect compared to $\mathrm{RH}$ parasites grown in human foreskin fibroblasts (HFFs) over 1 week (Fig. 1d). In contrast, $\triangle \mathrm{ACL} / \mathrm{i} \triangle \mathrm{ACS}$ parasites failed to form plaques after 7 days in the absence of Shld-1 (Fig. 1d), confirming our previous prediction of synthetic lethality [14]. Immunofluorescence assays (IFAs) revealed that $\mathrm{i} \triangle \mathrm{ACS}$ and $\triangle \mathrm{ACL}$ parasites were morphologically normal, while $\triangle \mathrm{ACL} / \mathrm{i} \triangle \mathrm{ACS}$ parasites presented a severe impairment in cell division with a loss of pellicle integrity as seen by clear alteration of staining of a pellicle marker, the gliding-associated protein 45 (GAP45) at $24 \mathrm{~h}$ after Shld-1 removal (Fig. 2a). Additionally, staining of the mitochondrion and apicoplast appeared diffuse in $\triangle \mathrm{ACL} / \mathrm{i} \triangle \mathrm{ACS}$ parasites, suggesting a loss of integrity of both organelles (Fig. 2b, c). Similarly, electron microscopy examination of $\triangle \mathrm{ACL} /$ i $\triangle$ ACS highlighted extreme morphological defects $24 \mathrm{~h}$ after Shld-1 removal (Fig. 2d, e). Some dividing $\triangle \mathrm{ACL} /$ i $\triangle \mathrm{ACS}$ parasites presented loss of the basal structure (Fig. 2e, top panel), or were entirely amorphic, with the contents of multiple fused parasites enclosed by a joined plasma membrane (Fig. 2e, bottom panel). Altogether, these results support our previous observation that ACL and ACS constitute a synthetic lethal pair, depletion of which causes arrest of parasite growth. 
a

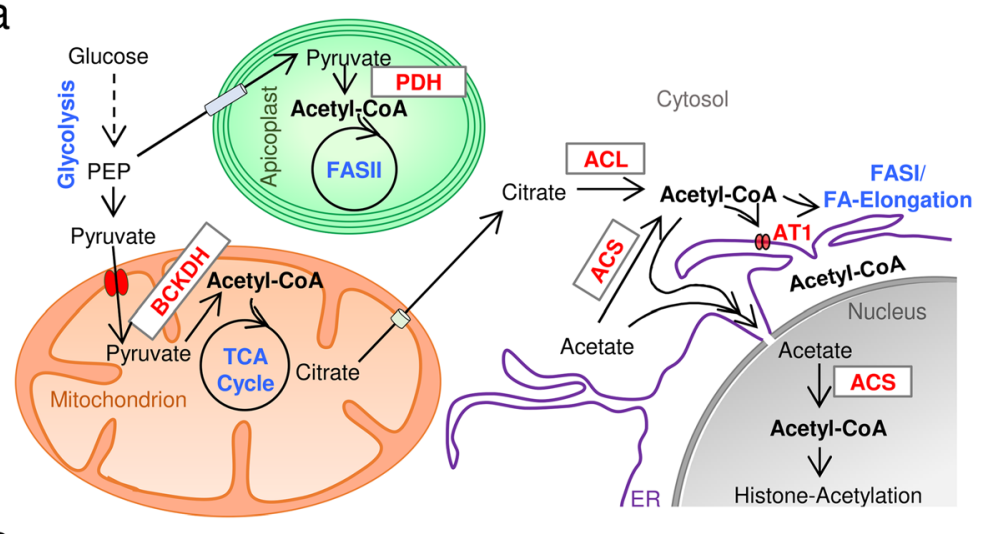

b

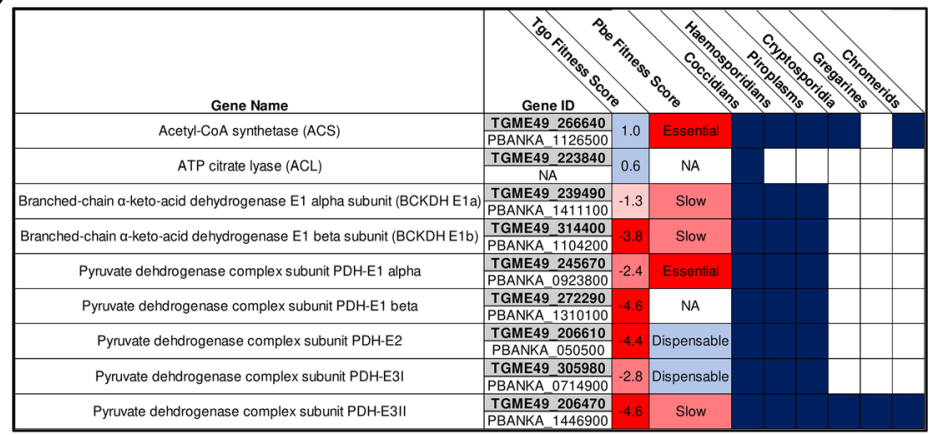

C

$\mathrm{i} \triangle \mathrm{ACS}$

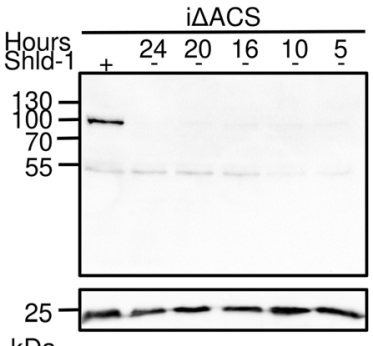

$\mathrm{kDa}$

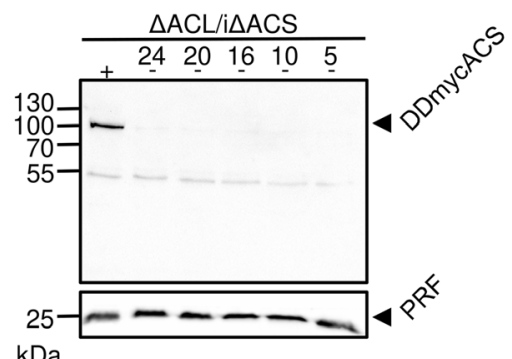

d

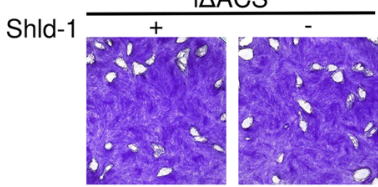

$\triangle \mathrm{ACL} / \mathrm{i} \triangle \mathrm{ACS}$

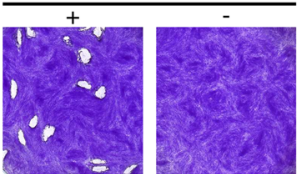

Fig. 1 ACS and ACL are essential to produce acetyl-CoA in the cytosol and nucleus. a Schematic representation of the metabolic pathways in $T$. gondii for acetyl-CoA production and transport into the cellular compartments where it is required: the apicoplast, mitochondrion, cytosol, nucleus and the endoplasmic reticulum (ER). Metabolic pathways are highlighted in blue and enzymes in red, and metabolites are depicted in black. BCKDH, branched-chain a-keto acid dehydrogenase-complex; PDC, pyruvate dehydrogenase complex; FA, fatty acid; FASII, type II FA synthase, ACL, ATP citrate lyase; ACS, acetyl-CoA synthetase; AT1, acetyl-CoA transporter; ER, endoplasmic reticulum; TCA, tricarboxylic acid. b Table highlighting the essentiality $[10,11]$ of acetyl-CoA-generating enzymes in T. gondii (Tgo) and Plasmodium berghei (Pbe) and their conservation across different apicomplexans. $\mathbf{c}$ Immuno-blot of total protein lysates from i $\triangle \mathrm{ACS}$ (left panel) and $\triangle \mathrm{ACL} / \mathrm{i} \triangle \mathrm{ACS}$ parasites (right panel) for which Shield-1 (Shld-1) was removed at several time points prior to egress to test protein regulation. Western blots were probed using a-myc antibody to detect the myc-tag of DD-ACS, and a-profilin (PRF) was used as a loading control. $\mathbf{d}$ Plaque assays were performed by inoculating human foreskin fibroblast (HFF) monolayers with either $\mathrm{I} \mathrm{ACS}$, or $\triangle \mathrm{ACL} / \mathrm{i} \triangle \mathrm{ACS}$ parasite strains and left to grow in the presence (+) or absence (-) of Shld-1 over a period of 7 days. Plaques were revealed by crystal violet staining of infected HFF monolayers 


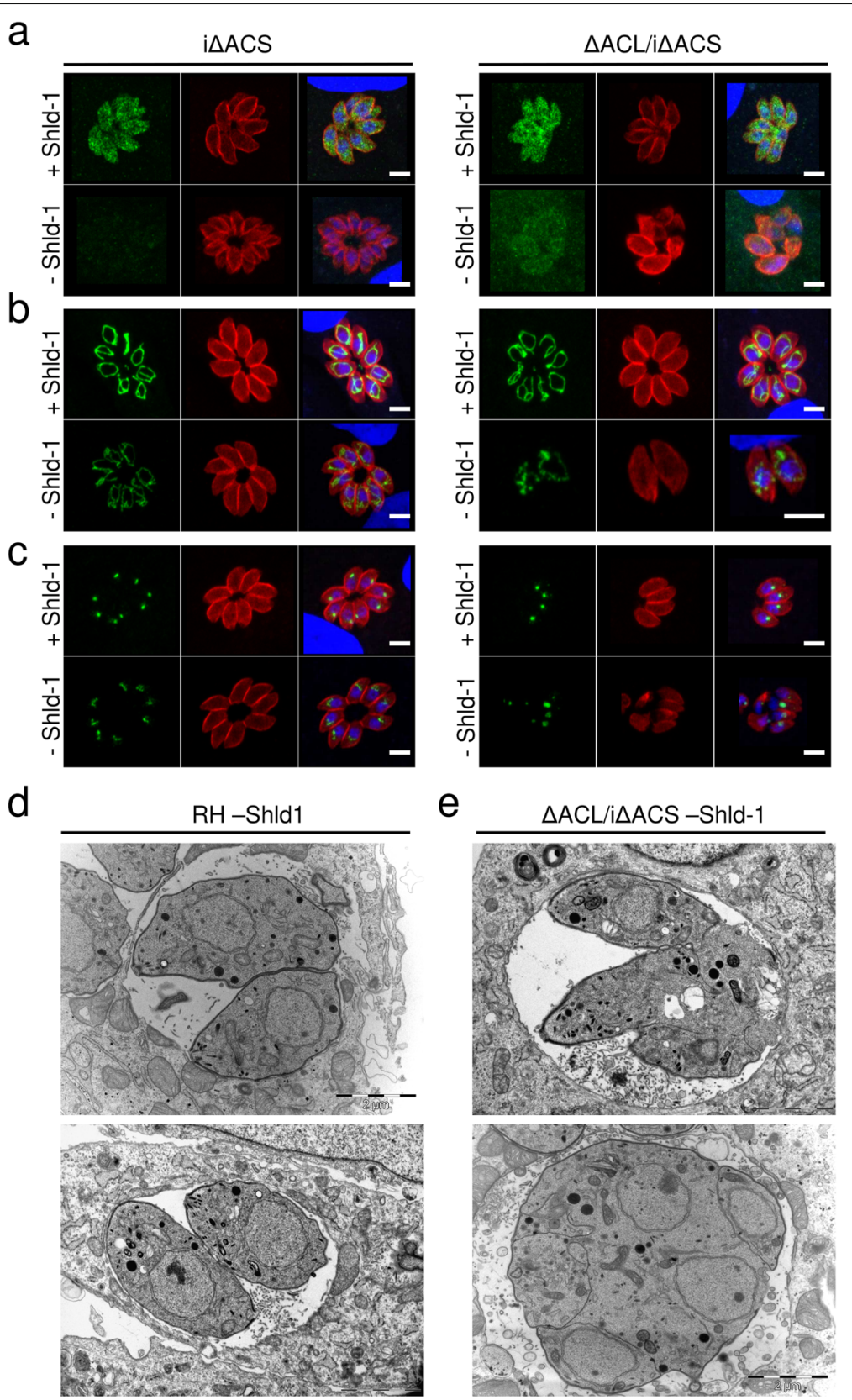

Fig. 2 Loss of ACS and ACL is associated with amorphic cells and loss of organelle integrity. Immunofluorescence assays (IFAs) of intracellular i $\triangle \mathrm{ACS}$ or $\triangle \mathrm{ACL} / \mathrm{i} \triangle \mathrm{ACS}$ parasites grown in the presence $(+)$ or absence $(-)$ of Shield-1 (Shld-1) for $24 \mathrm{~h}(\mathbf{a}-\mathbf{c})$. IFAs were fixed and stained with agliding-associated protein 45 (a-GAP45, red) to show pellicles of the parasites and 4,6 diamidin-2-phenylindol (DAPI, blue) to stain the nuclei and either, a-myc (green) to detect ACS (a) or with the monoclonal antibody 5F4 (a-F1B ATPase, green) marking the mitochondrion (b) or aapicoplast-associated thioredoxin family protein 1 (a-Atrx1, green) staining the apicoplast (c) (scale bars, $5 \mu \mathrm{m}$ ). Electron micrographs of intracellular $\mathrm{RH}$ (d) or $\triangle \mathrm{ACL} / \mathrm{i} \triangle \mathrm{ACS}$ (e) grown in the absence of Shld-1 for $24 \mathrm{~h}$ (scale bars, $2 \mu \mathrm{m})$. ACL, ATP citrate lyase; ACS, acetyl-CoA synthetase

\section{Lack of ACL/ACS alters the $T$. gondii metabolome including disruption of $F A$ elongation}

Analysing $\triangle \mathrm{ACL} / \mathrm{i} \triangle \mathrm{ACS}$ parasites by IFA and western blot allowed us to conclude that $16 \mathrm{~h}$ of Shld-1 removal was sufficient to deplete ACS in the $\triangle \mathrm{ACL} / \mathrm{i} \triangle \mathrm{ACS}$ strain, while parasites showed no growth defect or morphological abnormalities at this relatively early time point of ACS depletion and remained viable. In the following analyses, $\mathrm{i} \triangle \mathrm{ACS}$ and $\triangle \mathrm{ACL} / \mathrm{i} \triangle \mathrm{ACS}$ refer to parasites depleted of ACS by removal of Shld- 1 for $16 \mathrm{~h}$. To obtain a global picture of the impact of the loss of nucleo-cytosolic acetylCoA on parasite metabolism, we performed untargeted 
metabolomics using liquid chromatography-mass spectrometry (LC-MS). The full dataset is available in a data repository [24]. Over 850 putative metabolites were detected, and the relative abundance of all metabolites was compared to that of RH parasites (Additional file 2: Table S1). We focused our analysis on putative metabolites which changed more than 2 -fold in abundance ( $p$ value $<0.05$ ) and identified 40 putative metabolites as significantly perturbed, with 19 displaying decreased levels, while 21 were increased in abundance in $\triangle \mathrm{ACL} / \mathrm{i} \triangle \mathrm{ACS}$ parasites (Fig. 3a, Additional file 2: Table S1). While the loss of nucleo-cytosolic acetyl-CoA affected several metabolic pathways, many clustered into lipid/fatty acyl- and peptide metabolism (Additional file 2: Table S1, Additional file 3: Figure S2a). Enzymes producing or consuming the affected metabolites were almost exclusively predicted to localise to the cytosol or ER (Additional file 2: Table S1). Knock-out of ACL or knock-down of ACS alone had only minor impact with 14 or 1 putative metabolite changing, respectively (Additional file 2: Table S1). However, in many cases, loss of ACL alone led to a modest, non-significant drop in levels of certain metabolites, while the lack of both enzymes ( $\triangle \mathrm{ACL} /$ $\mathrm{i} \triangle \mathrm{ACS}$ ) aggravated the phenotype, indicating that the two enzymes have partially redundant functions in metabolism (Fig. 3b, Additional file 2: Table S1). Purine metabolites (adenine, inosine monophosphate (IMP), xanthine) were found increased in parasites lacking ACL and are likely associated with the HXGPRT resistance gene (Additional file 3: Figure S2a). Acetyl-CoA-levels decreased in $\triangle \mathrm{ACL}$ and $\triangle \mathrm{ACL} /$ i $\triangle$ ACS parasites to about $60 \%$ compared to $\mathrm{RH}$ cells, although the drop was not statistically significant (Fig. 3b). This indicates that the major pools of acetyl-CoA are in the mitochondrion and apicoplast, unaffected by the loss of ACS and ACL, consistent with the dramatic drop in total acetyl-CoA in $\triangle \mathrm{BCKDH}$ parasites [13].

Major changes were observed in the abundance of monounsaturated very long-chain FAs (FA C26:1, C28:1) by untargeted LC-MS analysis (Fig. 3b), consistent with acetyl-CoA being required for the elongation of FAs on the cytosolic site of the ER [25]. This defect in FA elongation in $\triangle \mathrm{ACL} / \mathrm{i} \triangle \mathrm{ACS}$ parasites was further scrutinised by semi-targeted profiling of FAs by gas chromatography-MS (GC-MS) (Fig. 3c). Using GC-MS, we found that the relative levels of five detected FAs were significantly altered between $\mathrm{RH}$ and $\triangle \mathrm{ACL} / \mathrm{i} \triangle \mathrm{ACS}$ parasites. While C18:0 and C20:4 were slightly increased in $\triangle \mathrm{ACL} / \mathrm{i} \triangle \mathrm{ACS}$ parasites, FA C18:1, C20:1 and C26:1 were significantly decreased, with FA C20:1 and C26:1 displaying a dramatic 2fold and 4-fold drop, respectively, while FA C28:1 was not detected by GC-MS (Fig. 3c). Importantly, most FAs are abundant in the host cells (HFFs) and can be salvaged by T. gondii. In particular, C18:0 and C20:4 make up a higher proportion of total FAs in the host compared to T. gondii [16]. Instead, C18:1 is of lower relative abundance in the host compared to T. gondii, and FAs C20:1, C26:1 and C28:1 are of very low abundance or absent in HFFs [16, 25]. We conclude that $T$. gondii $\triangle \mathrm{ACL} / \mathrm{i} \triangle \mathrm{ACS}$ compensate for the halt in FA elongation through increased uptake of unsaturated long-chain FAs from the host. The differences in FA abundances in HFFs compared to $T$. gondii [16] lead to the altered FA composition of $\triangle \mathrm{ACL} /$ $\mathrm{i} \triangle \mathrm{ACS}$ parasites. Additionally, we confirmed the defect in FA elongation by labelling with $\mathrm{U}_{-}{ }^{13} \mathrm{C}$-glucose or $\mathrm{U}_{-}{ }^{13} \mathrm{C}$ acetate for $16 \mathrm{~h}$ simultaneous to the ACS downregulation followed by GC-MS analysis. ${ }^{13} \mathrm{C}$-labelling in FA C26:1 was significantly decreased in $\triangle \mathrm{ACL} / \mathrm{i} \triangle \mathrm{ACS}$ parasites confirming the specific loss of FA elongation in these parasites (Fig. 3d, Additional file 3: Figure S2b). In contrast, the abundance as well as ${ }^{13} \mathrm{C}$-labelling in myristate (C14:0) was similar or increased in $\triangle \mathrm{ACL} / \mathrm{i} \triangle \mathrm{ACS}$ compared to $\mathrm{RH}$ parasites, indicating that cells are metabolically active and that de novo FA synthesis in the apicoplast is not affected (Fig. 3d, Additional file 3: Figure S2b). Possibly related to the reduced levels of long and very long monounsaturated FAs, abundance of the lipid phosphatidylserine (PS 38:2) was also found more than 2-fold reduced in $\triangle \mathrm{ACL} / \mathrm{i} \triangle \mathrm{ACS}$ parasites by LC-MS analysis (Fig. 3b). This may be a consequence of the altered abundance of C18:1 and C20:1, both of which were found to be significantly reduced in $\triangle \mathrm{ACL} / \mathrm{i} \triangle \mathrm{ACS}$ by semi-targeted GC-MS FA profiling, although the FA composition of the affected PS38:2 was not further scrutinised using tandem MS.

While the synthesis of monounsaturated very long chain FAs depends on acetyl-CoA as a substrate, the drop in levels of other metabolites such as D-fructose-1,6bisphosphate (F1,6-BP) is unexpected and may result from the altered acetylation status of enzymes in the pathway (Fig. 3b).

Additionally, we performed $\mathrm{U}_{-}{ }^{13} \mathrm{C}$-glucose and $\mathrm{U}_{-}{ }^{13} \mathrm{C}$ acetate labelling followed by LC-MS analysis, to compare the utilisation of these metabolites between $\mathrm{RH}$ and $\triangle \mathrm{ACL} /$ $\mathrm{i} \triangle \mathrm{ACS}$ parasites (Additional file 4: Figure S3a). We observed no differences in glucose utilisation when monitoring glycolytic and TCA cycle intermediates. While $\mathrm{U}_{-}{ }^{13} \mathrm{C}$ glucose labelling resulted in rapid and extensive labelling of glycolytic and TCA cycle intermediates, $\mathrm{U}-{ }^{13} \mathrm{C}$-acetate resulted in no or very little labelling of central carbon metabolites apart from citrate (Additional file 4: Figure S3b,c). Incubation in $\mathrm{U}_{-}{ }^{13} \mathrm{C}$-acetate resulted in $50 \%{ }^{13} \mathrm{C}$-labelling in the citrate of $\mathrm{RH}$, which was reduced more than 3-fold in $\mathrm{i} \triangle \mathrm{ACS}$ and $\triangle \mathrm{ACL} / \mathrm{i} \triangle \mathrm{ACS}$ parasites (Additional file 4: Figure S3c). The lack of/low levels of ${ }^{13} \mathrm{C}$-labelling in other TCA cycle intermediates indicate that the labelling observed in citrate is not in the mitochondrial pool but rather in the cytosolic or a putative apicoplast pool. We propose that a second citrate synthase 2, for which the localisation is yet unknown [26], condenses acetyl-CoA and oxaloacetate (OAA) to form citrate in the cytosol utilising acetyl- 
a

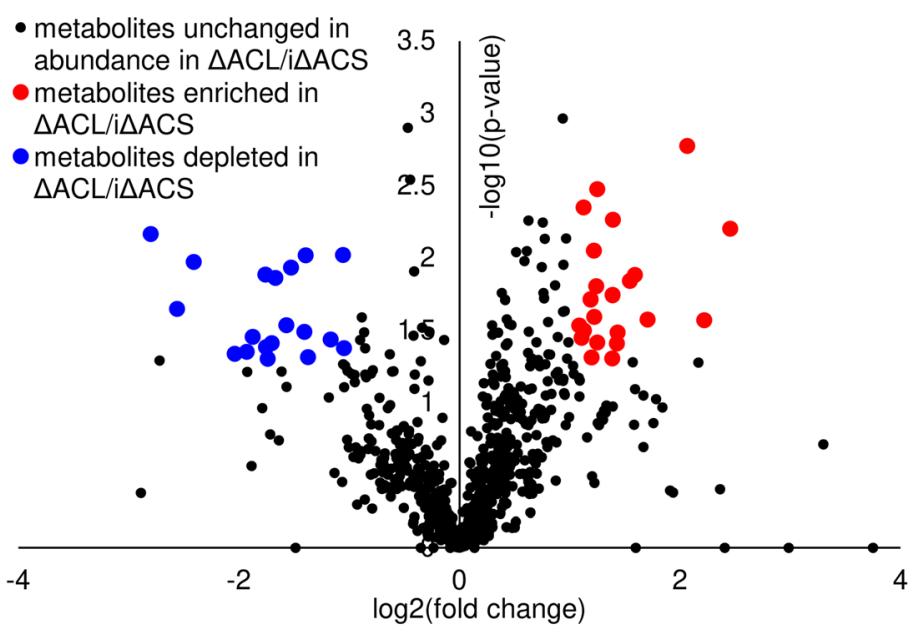

b

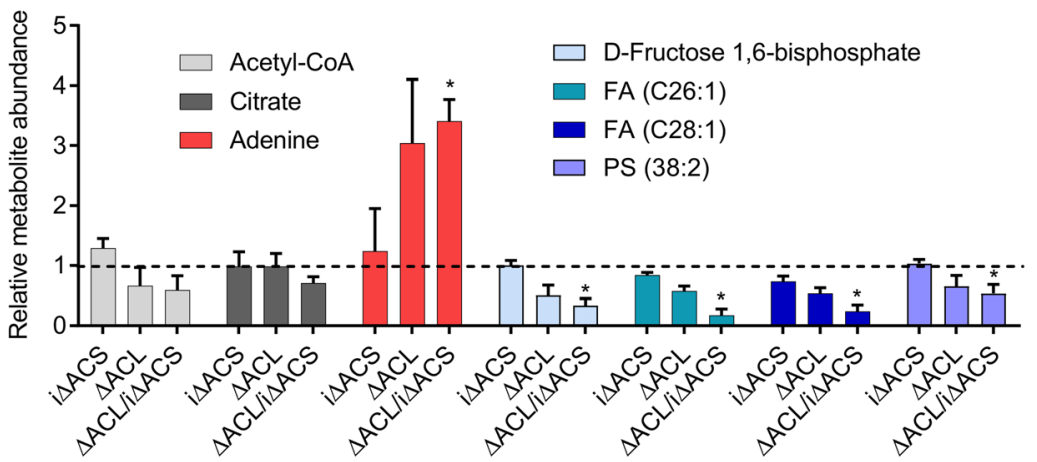

C

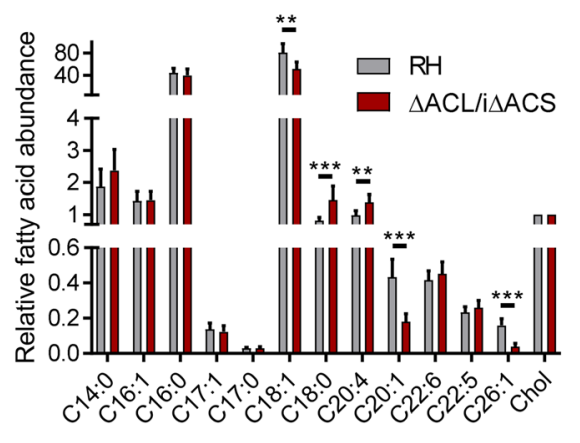

d

吕 Unlabelled fraction

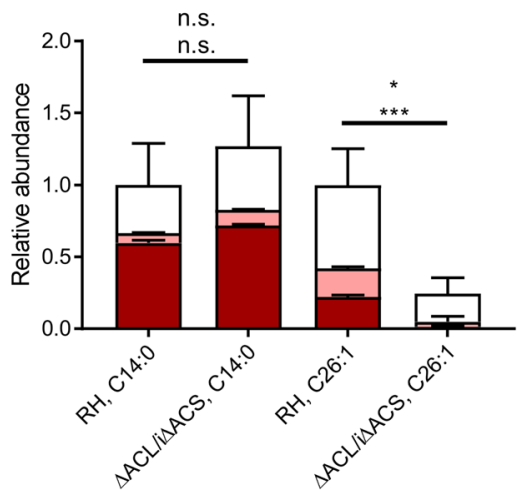

Fig. 3 (See legend on next page.) 


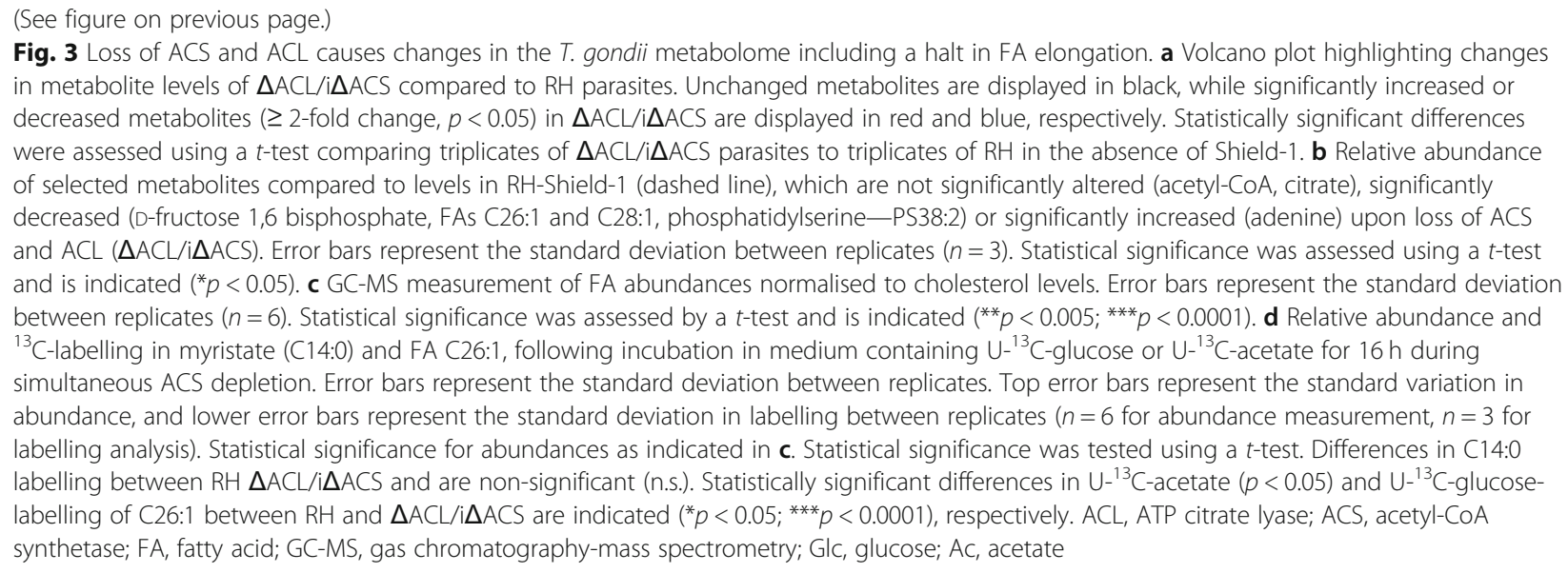

CoA generated by ACS which is derived from $\mathrm{U}_{-}{ }^{13} \mathrm{C}$-acetate. We also observed a significant decrease in labelling from $\mathrm{U}_{-}{ }^{13} \mathrm{C}$-acetate in some lipid species (Additional file 4: Figure S3d-f) including PS38:2 (Additional file 4: Figure S3f) which was also 2-fold reduced in abundance in $\triangle \mathrm{ACL} /$ i $\triangle$ ACS parasites (Fig. 3b). Overall, our metabolomic analyses demonstrate that lack of ACL/ACS results in several changes in the metabolism, most notably the loss of long and very long monounsaturated FAs, which have previously been demonstrated to be essential for $T$. gondii [25].

\section{Lack of ACL/ACS or BCKDH results in hypo-acetylation of cytosolic and mitochondrial proteins, respectively}

To determine the role of cytosolic and mitochondrial acetyl-CoA in protein acetylation, we characterised the acetylome of parasites lacking ACL and ACS or BCKDH, the complex implicated in the production of acetyl-CoA in the mitochondrion [13]. Generation of a parasite line lacking the $B C K D H$ subunit E1 $(\triangle B C K D H)$ was described previously [13]. Western blot analysis using $\alpha-$ acetyl-lysine antibodies revealed widespread Ne-lysineacetylation in $T$. gondii $\mathrm{RH}, \triangle \mathrm{ACL} / \mathrm{i} \triangle \mathrm{ACS}$ and $\triangle \mathrm{BCKDH}$ parasites (Additional file 5: Figure S4a,b). Quantitative MS-based proteomic analyses comparing $\mathrm{RH}$ and $\triangle \mathrm{ACL} / \mathrm{i} \triangle \mathrm{ACS}$ parasites allowed us to confidently quantify 404 acetylated sites on 269 proteins. Out of these, 182 sites (45\%) belonging to 137 proteins were found to be differentially acetylated in $\triangle \mathrm{ACL} / \mathrm{i} \Delta \mathrm{ACS}$ parasites compared to RH (Additional file 6: Table S2). Most sites (142) were hypo-acetylated while 40 were hyperacetylated in $\triangle \mathrm{ACL} / \mathrm{i} \triangle \mathrm{ACS}$ parasites (Fig. 4a). The predominant hypo-acetylation ( $78 \%$ of differentially acetylated sites) is consistent with the expected reduction of acetyl-CoA. The complete dataset is available in a data repository [28].

To determine the sub-cellular localisation of differentially acetylated proteins, we predicted their putative localisation based on the hyperplexed Localisation of
Organelle Proteins by Isotopic Tagging (hyperLOPIT) data available under https://proteome.shinyapps.io/toxolopittzex/ and on ToxoDB (https://toxodb.org) [27]. For simplification, we merged some of the sub-cellular compartments (19S proteasome/20S proteasome/40S ribosome/60S ribosome/cytosol into cytosol; nucleolus/ nucleus - chromatin/nucleus non-chromatin into nucleus and mitochondrion membranes/mitochondrion soluble into mitochondrion etc.). Differentially acetylated sites were found within proteins of several sub-cellular compartments; however, most hypo-acetylated residues (77\%) were of proteins within the cytosol and nucleus, consistent with the localisation of ACS and ACL (Fig. 4b). To account for the different number of proteins within the distinct sub-cellular compartments, we determined the number of differentially acetylated proteins relative to the total number of proteins identified within the compartment, which confirmed that hypo-acetylated proteins were enriched in the cytosol (12\% of all cytosolic proteins hypo-acetylated) and nucleus (4.5\% of all nuclear proteins hypo-acetylated) (Additional file 5: Figure S4c). Surprisingly, the few sites which were differentially acetylated on proteins in the apicoplast or mitochondrion in $\triangle \mathrm{ACL} / \mathrm{i} \triangle \mathrm{ACS}$ parasites were predominantly hyperacetylated (Fig. 4b). The affected hyper-acetylated apicoplast proteins are the $\mathrm{PDH}$, the enoyl-acyl carrier protein reductase (ENR) and a putative chaperone (Additional file 6: Table S2), while the affected mitochondrial proteins are putative chaperones, heat-shock proteins and TCA cycle enzymes (Additional file 6: Table S2). Perhaps, these proteins are hyper-acetylated as part of a stress response to avert the phenotype caused by loss of ACS and ACL. However, the effect of acetylation of these proteins is unknown, and hence, the functional consequences of their hyperacetylation remain unclear. While $\triangle \mathrm{ACL} / \mathrm{i} \triangle \mathrm{ACS}$ parasites showed deformation at $24 \mathrm{~h}$ of Shld- 1 removal, we argue that the observed hypo-acetylation observed in $\triangle \mathrm{ACL} /$ i $\triangle$ ACS parasites at $16 \mathrm{~h}$ of Shld- 1 removal is a direct result 


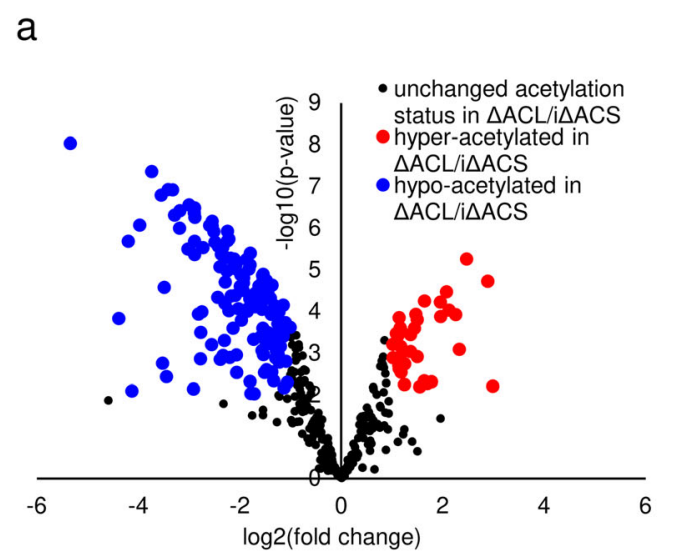

C

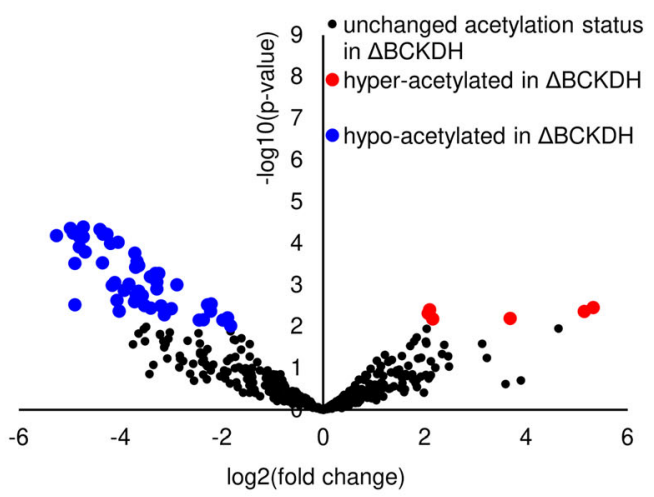

$\mathrm{b}$

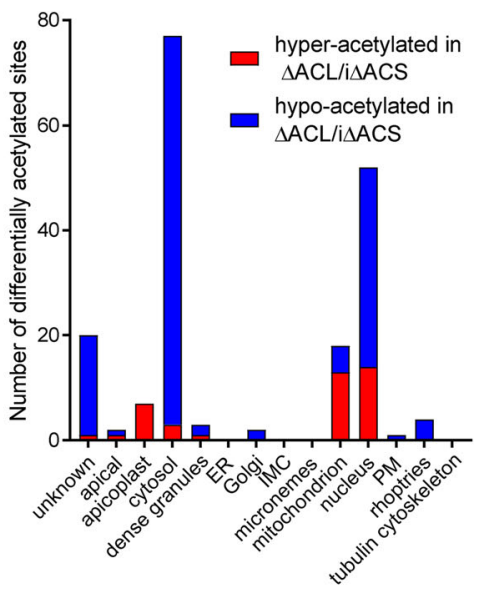

d

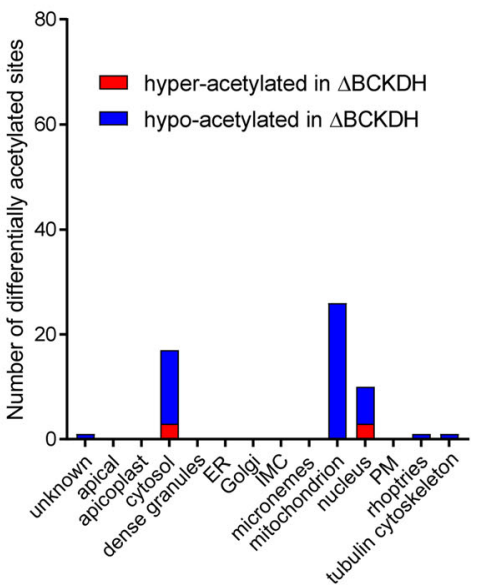

Fig. 4 Loss of ACL/ACS or BCKDH results in predominant hypo-acetylation of nucleo-cytosolic and mitochondrial proteins, respectively. a Volcano plot highlighting differentially acetylated sites of $\triangle A C L / i \triangle A C S$ compared to RH parasites. Statistically significant differences between parasite lines were determined as outlined in the 'Material and methods' section. Unchanged acetylation sites are displayed in black, while sites which are significantly hyper- or hypo-acetylated in $\triangle \mathrm{ACL} / \mathrm{i} \Delta \mathrm{ACS}(\geq 2$-fold, $n=3$, limma $p<0.01)$ are displayed in red and blue, respectively. $\mathbf{b}$ Hyper- and hypo-acetylated sites in $\triangle \mathrm{ACL} / \mathrm{i} \triangle \mathrm{ACS}$ parasites were sorted according to their sub-cellular localisation using the hyperplexed Localisation of Organelle Proteins by Isotopic Tagging (hyperLOPIT) data available under https://proteome.shinyapps.io/toxolopittzex/ [27]. c Volcano plot highlighting the differentially acetylated sites in $\triangle B C K D H$ compared to $\mathrm{RH}$ parasites. Statistically significant differences were determined as outlined in the 'Material and methods' section. Unchanged acetylation sites are displayed in black, while sites which are significantly ( $\geq 2$-fold, $n=3$, limma $p<0.01$ ) hyper- or hypo-acetylated in $\triangle B C K D H$ are displayed in red and blue, respectively. $\mathbf{d}$ Hyper-and hypo-acetylated sites in $\triangle \mathrm{BCKDH}$ parasites were sorted according to their sub-cellular localisation using hyperLOPIT data available under https://proteome.shinyapps.io/ toxolopittzex/ [27]. BCKDH, branched-chain a-keto acid dehydrogenase-complex; ACL, ATP citrate lyase; ACS, acetyl-CoA synthetase; PM, plasma membrane; IMC, inner membrane complex; ER, endoplasmic reticulum

of the loss of acetyl-CoA in the cytosol, rather than a general death phenotype. This is supported by the fact that hypo-acetylation is predominantly observed in the affected cytosol, while other compartments show unaltered acetylation or even hyper-acetylation of proteins (Fig. 4b). Evidence that $\triangle \mathrm{ACL} / \mathrm{i} \triangle \mathrm{ACS}$ parasites are equally viable and metabolically active as $\mathrm{RH}$ parasites at $16 \mathrm{~h}$ of Shld- 1 removal can also be inferred from the above described metabolomic analyses: e.g. $\triangle \mathrm{ACL} / \mathrm{i} \triangle \mathrm{ACS}$ parasites show equal rates of FA de novo synthesis (see FA C14:0 levels and labelling in Fig. 3d) as well as equal levels and labelling of most central carbon metabolites (see Additional file 2: Table S1 and Additional file 4: Figure S3a).

As for $\triangle \mathrm{ACL} / \mathrm{i} \triangle \mathrm{ACS}$ parasites, we probed the acetylome of parasites lacking BCKDH using MS-based quantitative analyses. After stringent filtering, we identified 483 acetylation sites distributed over 300 proteins in $\mathrm{RH}$ and $\triangle B C K D H$ parasites (Additional file 7: Table S3). Fifty-six lysine residues belonging to 45 different proteins were found to be differentially acetylated in parasites lacking BCKDH compared to $\mathrm{RH}$, with only 6 sites displaying hyper-acetylation and 50 sites being hypo- 
acetylated (Fig. 4c). Using the hyper-LOPIT data, we identified that most hypo-acetylated residues (52\%) belonged to proteins residing within the mitochondrion, consistent with a decreased production of acetyl-CoA within this compartment due to loss of BCKDH catalytic activity (Fig. 4d, Additional file 5: Figure S4d). Taken together, these findings reveal that the lack of ACL/ACS or $\mathrm{BCKDH}$ results in predominant hypo-acetylation of numerous proteins within the respective compartment.

\section{Cytosolic and mitochondrial acetyl-CoA are required for extensive acetylation of glycolytic and TCA cycle enzymes, respectively}

To better understand the consequences of the differential acetylation in $\triangle \mathrm{ACL} / \mathrm{i} \triangle \mathrm{ACS}$ and $\triangle \mathrm{BCKDH}$ parasites, we aimed to identify the affected biological processes by performing Gene Ontology (GO) enrichment using the R-package topGO [29]. Comparing the differentially acetylated proteins to the entire genome/proteome of $T$. gondii, we established that the affected proteins cluster into 12 significantly enriched $(p<0.001)$ biological processes including histone acetylation (33-fold enrichment), chromatin organisation (9-fold enrichment), protein acetylation (19-fold enrichment) and carbohydrate metabolic processes (4-fold enrichment) (Fig. 5a). To avoid bias towards proteins which were identified as acetylated in this study, we performed the GO enrichment analysis of differentially acetylated proteins against the relatively small subset of 269 acetylated proteins as background (Additional file 5: Figure S4e). This analysis revealed the affected proteins to be significantly enriched $(p<0.05)$ in 2 biological processes, namely chromatin organisation and cellular protein metabolic processes. The latter also includes protein modification and is thus consistent with the enrichment of histone and protein acetylation identified in the analysis against the total genome/proteome.

Specifically, the proteins differentially acetylated in $\triangle \mathrm{ACL} / \mathrm{i} \triangle \mathrm{ACS}$ parasites included several histones $(\mathrm{H} 2 \mathrm{Bb}$, $\mathrm{H} 2 \mathrm{Bv}, \mathrm{H} 4, \mathrm{H} 2 \mathrm{AZ}$ ) as well as histone-modifying enzymes (histone arginine methyltransferase PRMT1, histone lysine acetyltransferase MYST-A/B, GCN5-A/B, histone acetyltransferase subunit nua4 protein) (Additional file 6: Table S2) [30-33]. Furthermore, we observed considerable hypo-acetylation of the apicomplexan Apetala 2 (AP2) transcription factors AP2XII-4, AP2VIIa-7, AP2IX-7 and AP2IX-5 [34]. Interestingly, two of the differentially acetylated transcription factors, AP2XII-4 and AP2IX-7, have previously been shown to interact with GCN5-B, which is essential for replication [33]. Taken together, these changes in acetylation of histones, histone-modifying enzymes and transcription factors are expected to affect gene expression broadly. Cobbold et al. have previously reported extensive acetylation of
AP2 DNA binding proteins in P. falciparum, identifying 16 out of 28 AP2 factors to be acetylated in one or more positions [19]. T. gondii possesses 67 different AP2 transcription factors [34], 9 of which we identified to be acetylated in one or more positions (Additional file 6: Table S2). AP2XII-4 displayed extensive acetylation in 6 different sites, 3 of which were hypo-acetylated in $\triangle \mathrm{ACL} / \mathrm{i} \triangle \mathrm{ACS}$ parasites, while 2 were unchanged and one site was hyper-acetylated (Additional file 6: Table S2). In contrast, 2 acetylation sites were identified for its homologue in P. falciparum, PF3D7_0516800 [19]. Jeffers and Sullivan have previously identified 5 AP2 domain-containing proteins to be acetylated in $T$. gondii and similarly identified AP2XII- 4 to be extensively acetylated in 4 sites [20] compared to 6 sites we report here. A recent ground-breaking study identified the microrchidia (MORC) protein as a key regulator of $T$. gondii development by repressing sexual commitment [35]. Intriguingly, the authors found that MORC functions in a complex with AP2 transcription factors and recruits the histone deacetylase HDAC3 to repress genes which trigger sexual differentiation [35]. Thus, nucleo-cytosolic acetyl-CoA is essential for the regulation of T. gondii development by altering histone accessibility and presumably by modifying the activities and interactions of AP2 transcription factors through extensive acetylation.

Besides the hypo-acetylation of nucleo-cytosolic proteins, we also identified hypo-acetylation of four rhoptry proteins (ROP12, ROP17, ROP40 and RON2) and two dense granule proteins (GRA1 and GRA2) (Additional file 6: Table S2), indicating that acetylation of these secretory proteins relies on cytosolic acetyl-CoA, likely imported into the ER by AT-1 [14]. ROPs and GRAs are effector proteins secreted into the host cells during parasite invasion to hijack host cellular functions and to establish and modify the parasitophorous vacuole [36]. Jeffers and Sullivan similarly identified acetylation of ROP 17 and RON2 and proposed additionally acetylation of ROP8 and RON4 but did not detect acetylation of ROP12 [20]. If or how acetylation effects the function of these proteins unique to apicomplexan parasites remains unclear. Nevertheless, hypo-acetylation of these secretory proteins in $\triangle \mathrm{ACL} / \mathrm{i} \triangle \mathrm{ACS}$ parasites provides the first evidence that their acetylation relies on the cytosolic acetyl-CoA pool.

Next, we analysed the affected metabolic pathways by comparing the differentially acetylated proteins against the entire genome/proteome of T. gondii and using the metabolic pathway enrichment tool on ToxoDB (https:// toxodb.org/) based on the Kyoto Encyclopedia of Genes and Genomes (KEGG) database as the source. The highest metabolic pathway enrichment was found for proteins functioning in glycolysis/gluconeogenesis (9-fold). Most glycolytic/gluconeogenic enzymes were hypo- 


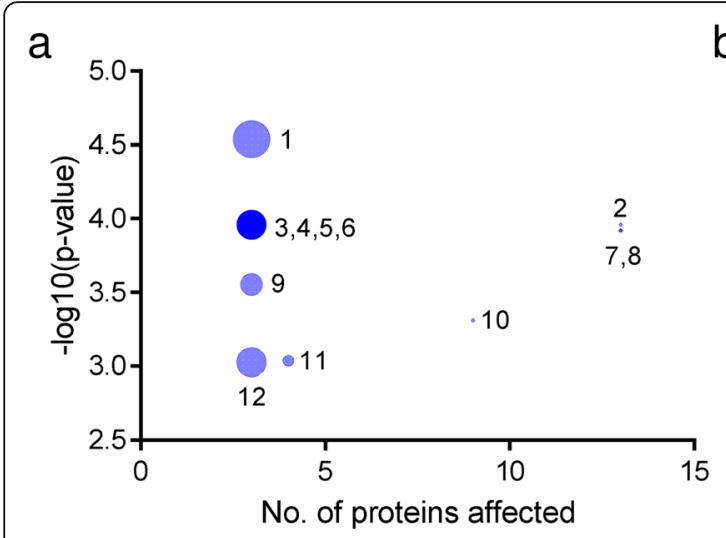

b

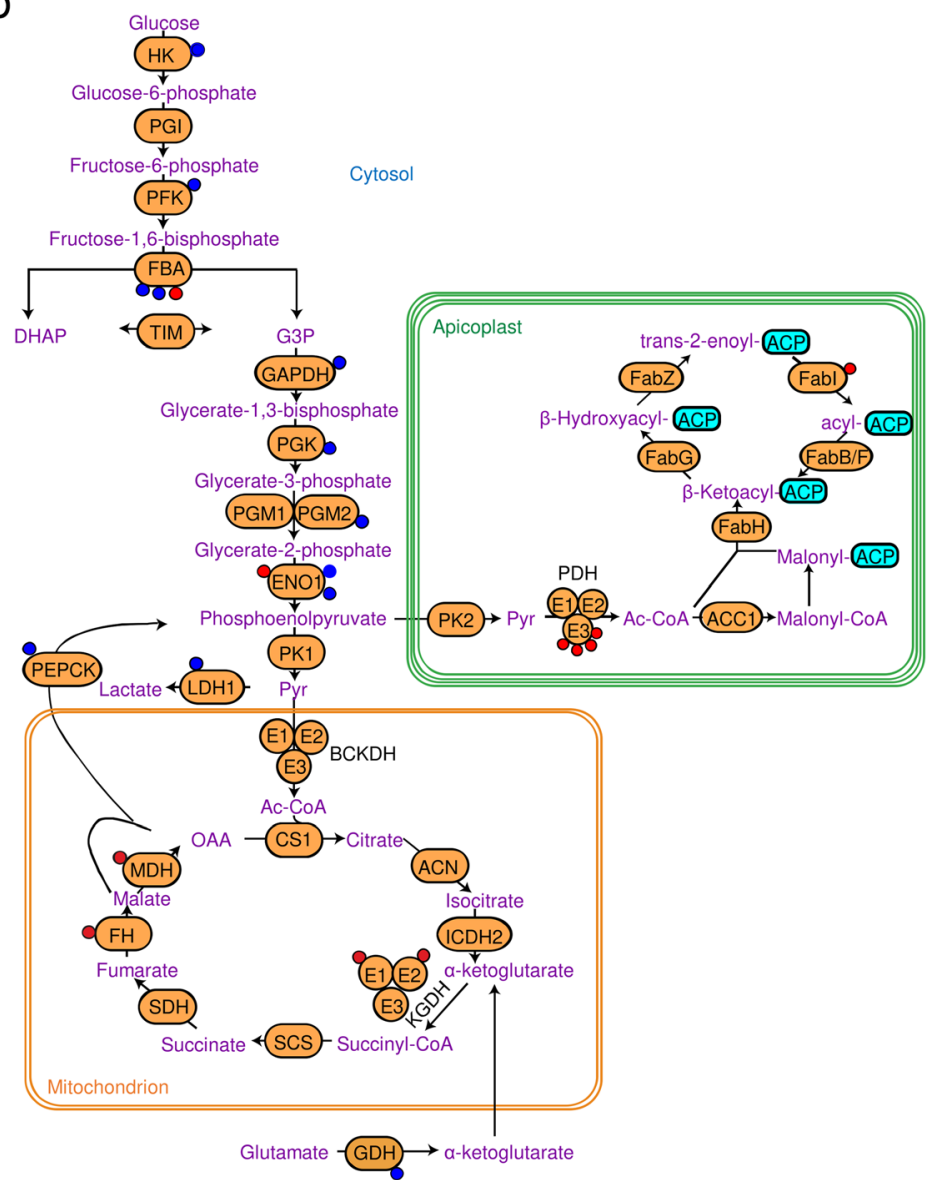

\begin{tabular}{|c|c|c|}
\hline No & GO-ID & \multicolumn{1}{|c|}{ Term } \\
\hline 1 & GO:0016573 & histone acetylation \\
\hline 2 & GO:0019752 & carboxylic acid metabolic process \\
\hline 3 & GO:0006473 & protein acetylation \\
\hline 4 & GO:0006475 & $\begin{array}{c}\text { internal protein amino acid } \\
\text { acetylation }\end{array}$ \\
\hline 5 & GO:0018393 & internal peptidyl-lysine acetylation \\
\hline 6 & GO:0018394 & peptidyl-lysine acetylation \\
\hline 7 & GO:0006082 & organic acid metabolic process \\
\hline 8 & GO:0043436 & oxoacid metabolic process \\
\hline 9 & GO:0043543 & protein acylation \\
\hline 10 & GO:0005975 & carbohydrate metabolic process \\
\hline 11 & GO:0006325 & chromatin organization \\
\hline 12 & GO:0018205 & peptidyl-lysine modification \\
\hline
\end{tabular}

Fig. 5 Loss of ACL/ACS causes extensive hypo-acetylation of glycolytic enzymes. a Proteins identified as differentially acetylated in $\triangle A C L / i \Delta A C S$ parasites were analysed using the GO enrichment R-package topGo to identify the enrichment in biological processes against the total $T$. gondii genome/proteome. The bubble sizes are proportional to the fold enrichment, ranging from 33-fold (histone acetylation, 1) to 3-fold (organic acid metabolic process, 7). Statistically significant enrichment was assessed by Fisher's exact test ( $p$ value $<0.001)$. b Scheme highlighting the distribution of differentially acetylated sites on metabolic enzymes of $\triangle \mathrm{ACL} / \mathrm{i} \triangle \mathrm{ACS}$ parasites. Displayed pathways include the glycolysis/ gluconeogenesis, the TCA cycle and the apicoplast resident FASII. Coloured circles on the enzymes represent sites which are either hyper- (red) or hypo-acetylated (blue) in $\triangle \mathrm{ACL} / \mathrm{IACS}$. ACL, ATP citrate lyase; ACS, acetyl-CoA synthetase; GO, Gene Ontology; TCA, tricarboxylic acid; FASII, fatty acid synthase II; HK, hexokinase; PGI, phosphoglucose isomerase; PFK, phosphofructokinase; FBA, fructose bis-phosphate aldolase; TIM, triosephosphate isomerase; GAPDH, glyceraldehyde 3-phosphate dehydrogenase; PGK, phosphoglycerate kinase; PGM, phosphoglycerate mutase; ENO, enolase; PK, pyruvate kinase; LDH, lactate dehydrogenase; PEPCK, phosphoenolpyruvate carboxykinase-1; BCKDH, branched-chain a-keto acid dehydrogenase-complex; E1-E3, BCKDH subunits; CS, citrate synthase; ACN, aconitase; ICDH, isocitrate dehydrogenase; KGDH, a-ketoglutarate dehydrogenase; E1-E3, KGDH subunits; SCS, succinyl coenzyme A synthetase; GDH, glutamate dehydrogenase; SDH, succinate dehydrogenase; FH, fumarate hydratase; MDH, malate dehydrogenase; PDH, pyruvate dehydrogenase complex; E1-E3, PDH subunits; Ac-CoA, acetyl-CoA; ACP, acyl carrier protein; Fab, FAS II subunits; ACP, acyl carrier protein; ACC, acetyl-CoA carboxylase; Pyr, pyruvate; OAA, oxaloacetate; DHAP,

dihydroxyacetone phosphate; G3P, glyceraldehyde-3-phosphate

acetylated in $\triangle \mathrm{ACL} / \mathrm{i} \triangle \mathrm{ACS}$ parasites (Fig. 5b). Although enzymes implicated in glycolysis are extensively acetylated in other organisms [37-39], the effects that acetylation has on enzyme activity varies and depends on the organism, enzyme, site and context [37-41]. In the metabolome analysis, we observed no differences in glycolytic flux, i.e. no changes in levels and labelling of most glycolytic intermediates (Additional file 2: Table S1, Additional file 4: Figure S3a), with the exception of a significant reduction in levels of $\mathrm{F} 1,6-\mathrm{BP}$ in $\triangle \mathrm{ACL} / \mathrm{i} \triangle \mathrm{ACS}$ parasites (Fig. 3b). Strikingly, the enzymes producing and consuming F1,6-BP, phosphofructokinase (PFK) and F1,6-BP aldolase (FBA), respectively, were both differentially acetylated in $\triangle \mathrm{ACL} / \mathrm{i} \triangle \mathrm{ACS}$ parasites. The differential acetylation may impact on the enzyme's activity and may cause reduced synthesis or increased consumption of F1,6-BP.

As described above for $\triangle \mathrm{ACL} / \mathrm{i} \triangle \mathrm{ACS}$ parasites, we performed the GO enrichment analysis of biological processes and metabolic pathways of proteins differentially 
a

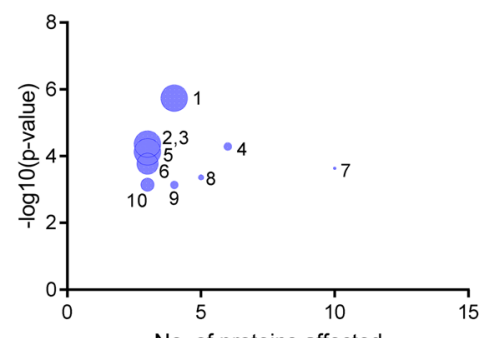

No. of proteins affected

\begin{tabular}{l|l|r}
\hline No & GO-ID & Term \\
\hline
\end{tabular}

1 GO:0072350

3 GO:0006101 citrate metabolic process

4 GO:0017144 drug metabolic process

5 GO:0009060 aerobic respiration

6 GO:0016999 antibiotic metabolic process

7 GO:0044281 small molecule metabolic process

8 GO:0006457 protein folding

9 GO:0045333 cellular respiration

10 GO:0006091 generation of precursor metabolites

c

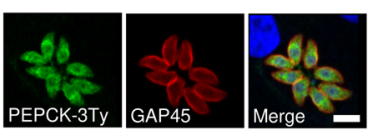

e

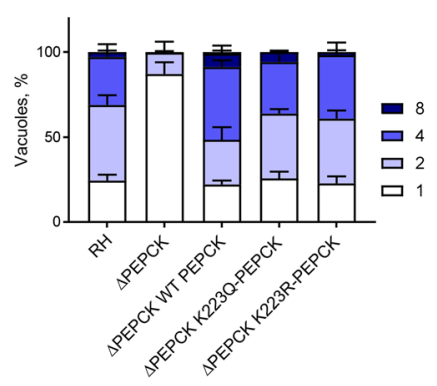

b

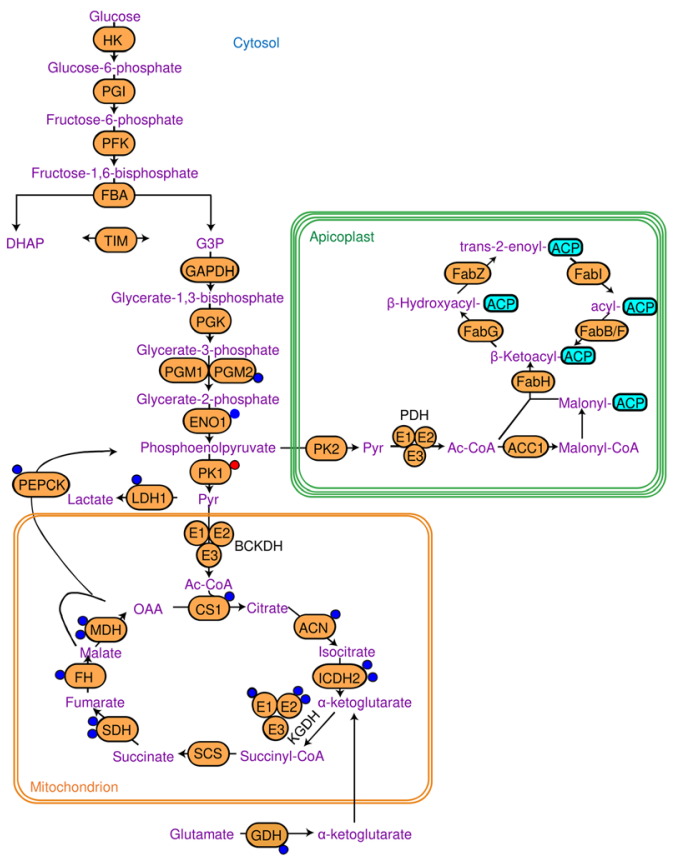

d

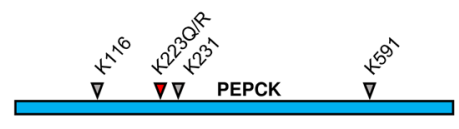

f

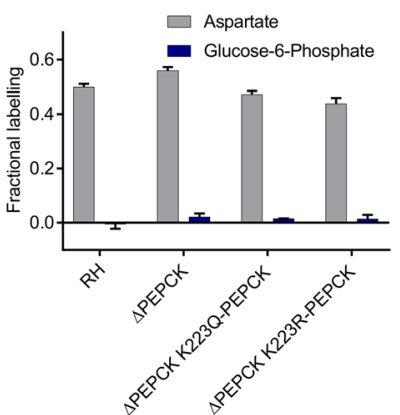

Fig. 6 (See legend on next page.) 
(See figure on previous page.)

Fig. 6 Loss of BCKDH causes extensive hypo-acetylation of TCA cycle enzymes and the gluconeogenic enzyme PEPCK-1. a Proteins identified as differentially acetylated in $\triangle \mathrm{BCKDH}$ parasites were analysed using the $\mathrm{GO}$ enrichment R-package topGo to identify enrichment in biological processes against the total $T$. gondii genome/proteome. The bubble sizes are proportional to the fold enrichment of the respective pathway, ranging from 36-fold (GO:0072350 — tricarboxylic acid metabolic process) to 4-fold (GO:0044281 —small molecule metabolic process). Statistically significant enrichment was assessed by Fisher's exact test ( $p$ value $<0.001$ ). b Scheme highlighting the distribution of differentially acetylated sites on metabolic enzymes of $\triangle B C K D H$ parasites. Displayed pathways include the glycolysis/gluconeogenesis, the TCA cycle and the apicoplast resident FASII. Coloured circles on the enzymes represent sites which are either hyper- (red) or hypo-acetylated (blue) in $\triangle B C K D H$. c Endogenous PEPCK-1 presents a nucleo-cytosolic localisation by immunofluorescence assay (IFA) after C-terminal tagging by knock-in of the endogenous PEPCK-1 locus both in RH parasites. PEPCK-1-3Ty was detected using a-Ty (green) while a-GAP45 (red) was used as a pellicle marker and 4',6 diamidin-2-phenylindol (DAPI, blue) to stain the nuclei (scale bars in $\mathbf{b}$ and $\mathbf{c}, 5 \mu \mathrm{m}$ ). $\mathbf{d}$ Schematic representation of PEPCK and its identified acetylation sites. Lysine at position K223 was changed to glutamine (K223Q acetylation mimetics) or arginine (K223R, de-acetylation mimetics). e The ability of different PEPCK-1 acetylation mimetics to grow in a medium lacking glucose was tested in an intracellular growth assay. Error bars represent the standard deviation between 3 independent infections. Per infection, > 100 vacuoles were counted. f Constitutive activation

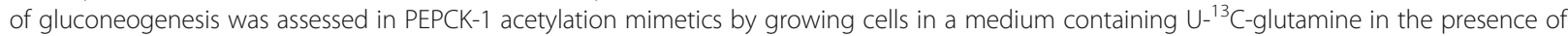
unlabelled glucose and measuring ${ }^{13} \mathrm{C}$-labelling in glycolytic intermediates using GC-MS (shown here, glucose-6-phosphate). Uptake and utilisation of U- ${ }^{13} \mathrm{C}$-glutamine were confirmed by measuring ${ }^{13} \mathrm{C}$-labelling in the TCA cycle by-product aspartate. Error bars represent the standard deviation between replicates $(n=3)$. Throughout the figure, PEPCK refers to PEPCK-1, the active enzyme in tachyzoites [42]. GO, Gene Ontology; TCA, tricarboxylic acid; FASIl, fatty acid synthase II; PEPCK, phosphoenolpyruvate carboxykinase-1; BCKDH, branched-chain a-keto acid dehydrogenase-complex; GC-MS, gas chromatography-mass spectrometry; TCA, tricarboxylic acid; other abbreviations, see Fig. 5

acetylated in $\triangle \mathrm{BCKDH}$ parasites. Differentially acetylated proteins in $\triangle \mathrm{BCKDH}$ parasites were compared to the total genome/proteome using the R-package topGO [29], which identified affected proteins to be significantly enriched $(p<0.001)$ in 10 biological processes, most of which are related to the TCA cycle and respiration, including the top hits with over 30-fold enrichment (tricarboxylic acid metabolic processes, tricarboxylic acid cycle and citrate metabolic processes) (Fig. 6a). In order to avoid bias towards acetylated proteins, differentially acetylated proteins were probed against the background of the small subset of acetylated proteins. Using this approach, 6 biological processes were identified as significantly enriched $(p<0.05)$ all of which related to the TCA cycle and respiration and 5 of which were also identified in the enrichment analysis against the entire genome/proteome (Additional file 5: Figure S4f). As expected, analysis of proteins which were differentially acetylated in $\triangle B C K D H$ parasites revealed major enrichment in the TCA cycle when using the metabolic pathway enrichment tool on ToxoDB (https://toxodb.org/) based on the KEGG database as the source, returned the TCA cycle as top-hit (24-fold enrichment). Seven out of eight TCA cycle enzymes were hypo-acetylated in $\triangle \mathrm{BCKDH}$ parasites compared to $\mathrm{RH}$ cells (Fig. 6b). As for glycolytic enzymes, the role of TCA cycle enzyme acetylation varies and has not been defined for $T$. gondii enzymes [41, 43].

One of the most significantly hypo-acetylated proteins in $\triangle \mathrm{BCKDH}$ cells was lactate dehydrogenase (LDH1) (Additional file 7: Table S3). Acetylation in lysine $\mathrm{K} 5$ has been reported to negatively regulate LDH activity in mammalian cells [44]. Although we observed hypo-acetylation of a different lysine (K218) in $\triangle \mathrm{BCKDH}$ parasites, it may similarly increase LDH activity, as lactate production is markedly increased in $\triangle \mathrm{BCKDH}$ parasites [13].

Furthermore, we revealed a previously unpublished acetylation site in the gluconeogenic enzyme phosphoenolpyruvate carboxykinase (PEPCK-1) (K116) in addition to the previously identified acetylation sites K223, K231 and K591 of T. gondii PEPCK-1 [20] (Additional file 7: Table S3). Interestingly, lysine residue K223 was found to be significantly hypo-acetylated in $\triangle B C K D H$ parasites (Additional file 7: Table S3, Fig. 6b). We previously reported constitutive activation of the gluconeogenesis pathway in $\triangle B C K D H$ parasites [13], leading us to hypothesise that the activation of gluconeogenesis in $\triangle \mathrm{BCKDH}$ parasites may be attributed to the changed acetylation status of PEPCK-1 (Additional file 8: Figure S5a).

\section{The PEPCK-1 acetylation status is not solely responsible for regulating gluconeogenesis in $T$. gondii}

In $T$. gondii, gluconeogenesis is tightly regulated and inactive under glucose replete conditions $[45,46]$ but essential in glucose-limiting conditions [42, 47]. T. gondii possess two PEPCKs, with PEPCK-1 (TGME49_289650) being the active enzyme in tachyzoites [42]. We identified two in-frame translation starts for PEPCK-1 (Additional file 8: Figure S5b). While the long isoform of PEPCK-1 localises to the mitochondrion (Additional file 8: Figure S5c), a second ATG, 285 bases downstream of the first predicted translational start, leads to a shorter isoform with cytosolic localisation (Additional file 8: Figure S5c). Crucially, a 3-Ty-epitope tag at the C-terminal end of the endogenous gene locus (Additional file 8: Figure S5d,e) revealed cytosolic localisation of the endogenous PEPCK-1 (Fig. 6c), contrasting previous reports, which have proposed a mitochondrial localisation [42]. To test 
whether the constitutive activation of gluconeogenesis in $\triangle B C K D H$ parasites [13] is due to hypo-acetylation of PEPCK-1 K223, we complemented $\triangle$ PEPCK-1 parasites with wild-type PEPCK-1 or versions of PEPCK-1 mimicking acetylation (glutamine) or de-acetylation (arginine) of lysine at position 223 (K223Q, K223R) (Fig. 6d). The pepck-1 locus was deleted by double homologous recombination (Additional file 8: Figure S5f). Its deletion and insertion of the chloramphenicol acetyltransferase (CAT) resistance cassette were confirmed by genomic PCR (Additional file 8: Figure S5g). Expression of the acetylation mimetic PEPCK-1 constructs was confirmed by IFA (Additional file 8: Figure S5h).

To investigate whether gluconeogenesis was constitutively active or inactive depending on the PEPCK-1 acetylation status, $\triangle$ PEPCK-1 strains complemented with PEPCK-1 acetylation mimetics (K223Q, K223R) were assessed in a growth assay in glucose-depleted medium (Fig. 6e). The acetylation/de-acetylation mimetics of PEPCK-1 (K223Q/ K223R) grew equally well and comparable to parasites complemented with WT-PEPCK-1 in glucose-depleted medium (Fig. 6e). Only parasites lacking PEPCK-1 showed a growth defect in the glucose-depleted medium. These findings indicate that acetylation of PEPCK-1 (K223Q) alone is not sufficient to de-activate gluconeogenesis. To assess the constitutive activation of gluconeogenesis, PEPCK-1 acetylation mimetic parasites were incubated in a medium containing unlabelled glucose and $\mathrm{U}_{-}{ }^{13} \mathrm{C}$-glutamine followed by profiling of polar metabolites by GC-MS (Fig. 6f). Labelled carbons from $\mathrm{U}_{-}{ }^{13} \mathrm{C}$-glutamine were not incorporated into glycolytic/gluconeogenic intermediates, such as glucose-6phospate, in the presence of unlabelled glucose (Fig. 6f), as was previously observed in $\triangle B C K D H$ parasites [13], highlighting that activation of gluconeogenesis is not triggered by de-acetylation of PEPCK-1 K223 (K223R) alone. Hence, the impact of acetylation on the function of enzymes in $T$. gondii central carbon metabolism remains unclear. Lastly, we assessed whether gluconeogenesis was an important adaptation in parasites lacking BCKDH. As described above for $\mathrm{RH}$, pepck-1 was deleted in parasites lacking BCKDH (Additional file 8: Figure S5i). Strikingly, $\triangle \mathrm{BCKDH} / \triangle \mathrm{PEPCK}-1$ parasites showed no aggravation of their growth phenotype compared to parasites lacking either BCKDH or PEPCK- 1 as assessed by plaque assay and intracellular growth assay in the presence and absence of glucose (Additional file 8: Figure S5j-1). These results highlight that the activation of gluconeogenesis in $\triangle \mathrm{BCKDH}$ parasites is a 'side effect' rather than a crucial adaptation mechanism.

\section{Lack of ACL/ACS or BCKDH alters the transcriptome and proteome of $T$. gondii}

For a global assessment of the impact caused by the depletion of cytosolic acetyl-CoA on gene expression in $\triangle \mathrm{ACL} /$ $\mathrm{i} \triangle \mathrm{ACS}$ parasites, we performed a combination of expression profiling by ribonucleic acid sequencing (RNA-seq) and quantitative proteomics analyses on freshly egressed extracellular tachyzoites. The complete datasets are available in data repositories $[28,48]$. We focused our analysis on transcripts and proteins that were changed $\geq 2$-fold in their level $(p$ value $<0.01)$ in $\triangle \mathrm{ACL} / \mathrm{i} \triangle \mathrm{ACS}$ parasites compared to RH. RNA-seq analysis allowed the identification of 453 differentially regulated transcripts out of 7250; 377 were found to be upregulated while 76 were downregulated (Fig. 7a, Additional file 9: Table S4). The predominant upregulation of transcripts in parasites depleted in nucleo-cytosolic acetyl-CoA was surprising, considering that histone de-acetylation is associated with a general downregulation of transcription [7]. As anticipated, the HXGPRT resistance cassette transcript belonged to the top upregulated hits while ACL was amongst the top hits of downregulated transcripts, validating the obtained results. Transcripts or proteins influenced directly by the experimental procedure were excluded from further analysis. Of the differentially acetylated transcripts, 284 encoded hypothetical proteins, hindering interpretation of the results. The remaining transcripts encoded for proteins of diverse functions and included several T. gondiispecific proteins such as surface antigen (SAG)-related proteins (16 transcripts) and Toxoplasma gondii family (A, $\mathrm{B}, \mathrm{C})$ proteins, a group of uncharacterised $T$. gondii-specific proteins (14 transcripts). GO enrichment against the entire genome/proteome of $T$. gondii using the biological process enrichment tool on ToxoDB (https://toxodb.org/) revealed no significant enrichment of the affected transcripts in biological processes.

MS-based proteomics allowed identification and relative quantification of $2773 \mathrm{~T}$. gondii proteins, out of which 74 were found to be differentially expressed in $\triangle \mathrm{ACL} / \mathrm{i} \triangle \mathrm{ACS}$ parasites compared to $\mathrm{RH}$ (Fig. 7a, Additional file 10: Table S5). In contrast to the transcriptome, most proteins [49] were downregulated in $\triangle \mathrm{ACL} / \mathrm{i} \Delta \mathrm{ACS}$ parasites (Fig. 7a, Additional file 10: Table S5). Amongst the downregulated proteins were ACS and ACL, while the HXGPRT selection cassette was amongst the top hits of upregulated proteins, validating the obtained data (Additional file 10: Table S5). The altered proteins have highly variable functions and include 26 hypothetical proteins, hindering the interpretation of the results.

Myosin J (MyoJ), a myosin motor mediating constriction of the basal pole during parasite division [50], was found to be downregulated over 2 -fold ( $p$ value $<0.01$ ) at the protein level in $\triangle \mathrm{ACL} / \mathrm{i} \triangle \mathrm{ACS}$ strain compared to RH (Additional file 10: Table S5). Loss of MyoJ has been shown to be associated with a loss of fitness in T. gondii, resulting in asynchronous division and the loss of connection between daughter cells [50]. Hence, its downregulation may contribute to the severe morphological 
a

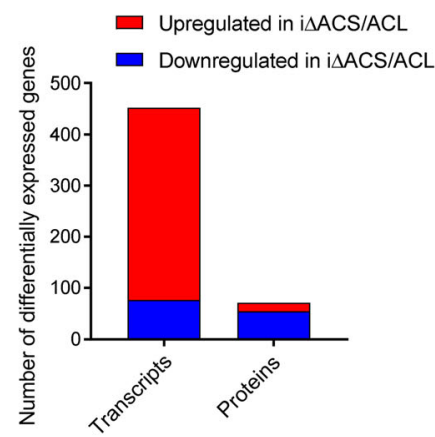

C

\begin{tabular}{|c|c|}
\hline \multicolumn{2}{|c|}{ Transcriptome/proteome } \\
\hline TGME49_294970 & hypothetical protein \\
\hline TGME49_237020 & exonuclease \\
\hline TGME49_308950 & $\begin{array}{l}\text { histidine acid phosphatase } \\
\text { superfamily protein }\end{array}$ \\
\hline TGME49_292280 & $\begin{array}{l}\text { SAG-related sequence } \\
\text { SRS36D }\end{array}$ \\
\hline TGME49_216140 & $\begin{array}{l}\text { tetratricopeptide repeat- } \\
\text { containing protein }\end{array}$ \\
\hline TGME49_273980 & hypothetical protein \\
\hline TGME49_229140 & $\begin{array}{l}\text { MaoC family domain-containing } \\
\text { protein }\end{array}$ \\
\hline TGME49_225790 & PDI family protein \\
\hline
\end{tabular}

e

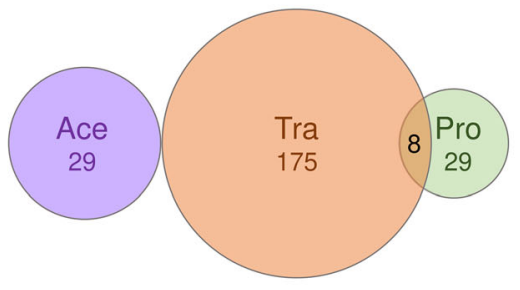

$f$ b

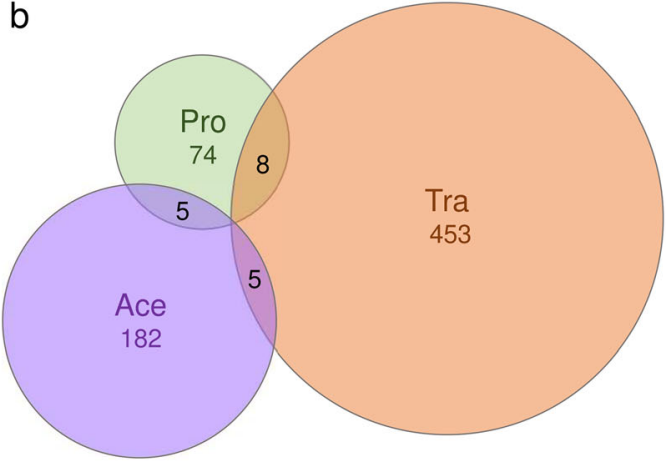

d

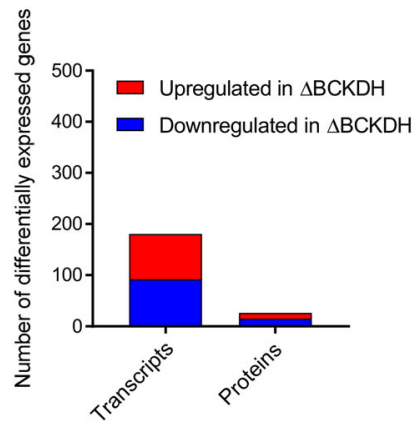

\begin{tabular}{|l|c|}
\hline \multicolumn{2}{|c|}{ Transcriptome/proteome } \\
\hline TGME49_209800 & formate/nitrite transporter protein \\
\hline TGME49_278390 & Toxoplasma gondii family A protein \\
\hline TGME49_305610 & hypothetical protein \\
\hline TGME49_229140 & MaoC family domain-containing protein \\
\hline TGME49_262050 & rhoptry kinase family protein ROP39 \\
\hline TGME49_320740 & hypothetical protein \\
\hline TGME49_215785 & rhoptry protein ROP2A \\
\hline TGME49_215775 & rhoptry protein ROP8 \\
\hline
\end{tabular}

Fig. 7 Loss of ACL/ACS or BCKDH alters the T. gondii gene expression. a Graph showing the number of RNA transcripts and proteins which are $\geq 2$-fold up- (red) or downregulated (blue) in $\triangle A C L / i \Delta A C S$ parasites compared to RH ( $n=3$; limma $p<0.01$ ). Statistical significance was determined as outlined in the 'Material and methods' section. $\mathbf{b}$ Venn diagram highlighting the overlap between differentially expressed RNA transcripts (Tra), proteins (Pro) and differentially acetylated proteins (Ace) which present a $\geq 2$-fold change in $\triangle \mathrm{ACL} / \mathrm{i} \triangle \mathrm{ACS}$ parasites compared to RH parasites (all $p<0.01$ ). c Table highlighting genes which were found to be significantly up- (red background) and downregulated (blue background) at the transcriptome and proteome level in $\triangle A C L / I A C S$ parasites. $\mathbf{d}$ Graph highlighting the number of RNA transcripts and proteins which are $\geq 2$-fold up- (red) or downregulated (blue) in $\triangle \mathrm{BCKDH}$ parasites compared to $\mathrm{RH}(n=3$; limma $p<0.01$ ). Statistical significance was determined as outlined in the 'Material and methods' section. e Venn diagram highlighting the overlap between differentially regulated acetylation sites (Ace), RNA transcripts (Tra) and proteins (Pro), which present a $>2$-fold change $(p<0.01)$ in $\Delta$ BCKDH parasites compared to RH parasites. $\mathbf{f}$ Table highlighting genes which were found to be significantly up- (red background) and downregulated (blue background) at the transcriptome and proteome level in $\triangle \mathrm{BCKDH}$ parasites. BCKDH, branched-chain a-keto acid dehydrogenase-complex; ACL, ATP citrate lyase; ACS, acetyl-CoA synthetase; RNA, ribonucleic acid

defects of $\triangle \mathrm{ACL} / \mathrm{i} \triangle \mathrm{ACS}$ parasites described above (Fig. 2).

A relatively small subset of 8 genes was affected simultaneously at the transcriptome and proteome level, with 2 genes being downregulated at the RNA transcript and protein level, while 6 were upregulated at both levels (Fig. 7b, c). This relatively small overlap between the datasets highlights the diverse and complex consequences of cytosolic acetyl-CoA depletion.

Similarly, expression profiling by RNA-seq and quantitative proteomics was performed on freshly egressed $\triangle \mathrm{BCKDH}$ tachyzoites to obtain a more holistic assessment of the consequences of mitochondrial acetyl-CoA depletion. Transcriptomic analysis revealed that a 
relatively small subset of transcripts (175 out of 6926) was differentially expressed ( $\geq 2$-fold change, $p$ value $<$ 0.01 ) in $\triangle B C K D H$ compared to $\mathrm{RH}$ parasites (Fig. $7 \mathrm{~d}$, Additional file 11: Table S6) with 90 transcripts being down- and 85 being upregulated. Upregulation of the HXGPRT resistance cassette transcripts and downregulation of BCKDH subunit E1 transcripts provided confidence in the results. Of the differentially expressed transcripts, 100 encode hypothetical proteins hindering the interpretation of the results. GO enrichment analysis using the biological process enrichment tool on ToxoDB (https://toxodb.org/) against the total T. gondii genome/ proteome indicated a significant enrichment of differentially expressed transcripts in cell differentiation $(p<0.05)$ although only 2 transcripts were affected (Additional file 11: Table S6). Many transcripts encode T. gondii-specific proteins such as SAG-related proteins (12 transcripts) and Toxoplasma gondii family proteins (6 transcripts) (Additional file 11: Table S6).

MS-based proteomics allowed identification and relative quantification of $2242 \mathrm{~T}$. gondii proteins. As for the transcriptome, a relatively small subset of proteins [30] was found to be differentially expressed ( $\geq 2$-fold change, $p$ value $<0.01)$ in $\triangle B C K D H$ parasites, out of which 16 were downregulated in the mutant parasites while 13 were upregulated (Fig. 7d, Additional file 12: Table S7). Downregulation of BCKDH-E1 and upregulation of HXGPRT validated the obtained data (Additional file 12: Table S7). The differentially expressed proteins included 8 hypothetical proteins and proteins of diverse functions. GO term enrichment analysis of biological processes (limited to GO slim terms) against the entire genome/ proteome of $T$. gondii using the tool on ToxoDB indicated significant enrichment $(p<0.05)$ in carbohydrate metabolic processes and cellular protein modification processes, with 3 and 4 proteins affected, respectively (Additional file 12: Table S7). Comparison of the different datasets revealed no overlap of the altered acetylome with either the transcriptome or proteome. However, 8 genes were differentially expressed simultaneously at the transcript and protein level (Fig. 7e, f), 3 of which were significantly upregulated at the transcript and protein level, while 5 were downregulated at both levels, including 3 rhoptry proteins, which are secreted during parasite invasion [36] (Fig. 7f).

One gene, for which expression was upregulated over 2fold at the transcript and protein levels in $\triangle \mathrm{BCKDH}$ parasites, belonged to the formate/nitrite transporter (FNT) family (TGME49_209800, FNT-1) (Fig. 7f). In coccidians, three members of this protein family catalyse the transport of monocarboxylate metabolites such as lactate (Fig. 8a) [51]. In contrast, haemosporidians and piroplasms express a single FNT, which has been shown to be essential in Plasmodium and is the target of several antimalarials [52-
54]. T. gondii parasites lacking $\mathrm{BCKDH}$ have previously been shown to have increased levels of intracellular pyruvate and lactate due to the disruption of the link between glycolysis and the TCA cycle [13]. Under these circumstances, lactate secretion may be increasingly important to maintain metabolic homeostasis.

\section{FNT-1 is dispensable in RH but becomes fitness- conferring in $\triangle \mathrm{BCKDH}$ parasites}

To assess whether the upregulation of FNT-1 is important for the metabolic adaptation in $\triangle B C K D H$ cells, we deleted fnt-1 in $\mathrm{RH}$ and $\triangle \mathrm{BCKDH}$ parasites. Using CRISPR-Cas9, we replaced the fnt-1 locus with a dihydrofolate reductase-thymidylate synthase (dhfr-ts) resistance cassette (Additional file 13: Figure S6a). Integration of the resistance cassette and the absence of the original gene locus were confirmed by PCR in the single- and double-knock-out (KO) (Additional file 13: Figure S6b). Assessment of the fitness of the different strains by plaque assay confirmed that $\triangle \mathrm{BCKDH}$ parasites present a modest but significant reduction in plaque size (Fig. $8 \mathrm{~b}$, c), as previously reported [13]. In contrast, $\Delta$ FNT-1 parasites formed plaques of normal sizes comparable to $\mathrm{RH}$ cells, while $\triangle B C K D H / \triangle F N T-1$ parasites formed very small plaques, which were considerably smaller than those of $\triangle \mathrm{BCKDH}$ cells (Fig. 8b,c). An intracellular growth assay confirmed the normal development of $\triangle$ FNT-1 cells and the aggravated phenotype of $\triangle$ BCKDH/ $\triangle$ FNT-1 compared to $\triangle$ BCKDH cells (Fig. 8d). An overview of the growth and fitness defects of the various strains analysed in this study is provided (Additional file 13: Figure S6c).

To characterise the defect in the metabolism of $\triangle \mathrm{BCKDH} / \triangle \mathrm{FNT}-1$ parasites, intracellular metabolites involved in central carbon metabolism were profiled by GC-MS (Fig. 8e). Interestingly, none of the detected metabolites was significantly altered in $\triangle \mathrm{FNT}-1$ parasites compared to $\mathrm{RH}$. Instead, $\triangle \mathrm{BCKDH}$ parasites displayed significant changes such as increased lactate and markedly reduced citrate consistent with our previous analysis [13]. $\triangle \mathrm{BCKDH} / \triangle \mathrm{FNT}-1$ presented an aggravation of the metabolic phenotype observed in $\triangle \mathrm{BCKDH}$ parasites (Fig. 8e). The observed 5-fold increase in intracellular lactate in $\triangle \mathrm{BCKDH} / \triangle \mathrm{FNT}-1$ (compared to 2-fold in $\triangle \mathrm{BCKDH})$ likely impacts on the parasite fitness [51, 53, 55]. To specifically test whether $\triangle \mathrm{FNT}-1$ and $\triangle \mathrm{BCKDH} /$ $\triangle$ FNT-1 parasites displayed defective lactate secretion, we measured the levels of metabolites secreted into the medium by the different $T$. gondii strains (Fig. 8f). Secreted metabolites detected in the medium included lactate, alanine, succinate, aspartate and glutamate, consistent with previous studies [46]. $\triangle \mathrm{BCKDH}$ cells displayed the highest secretion of lactate, while $\triangle \mathrm{FNT}-1$ secreted the lowest levels of lactate (Fig. 8f). $\triangle \mathrm{BCKDH} /$ 
a

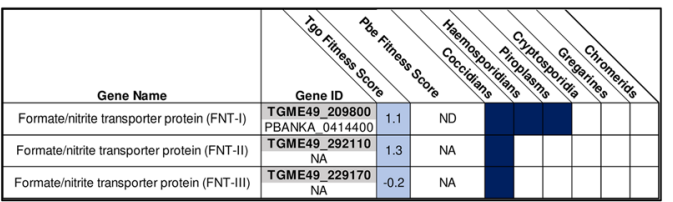

b
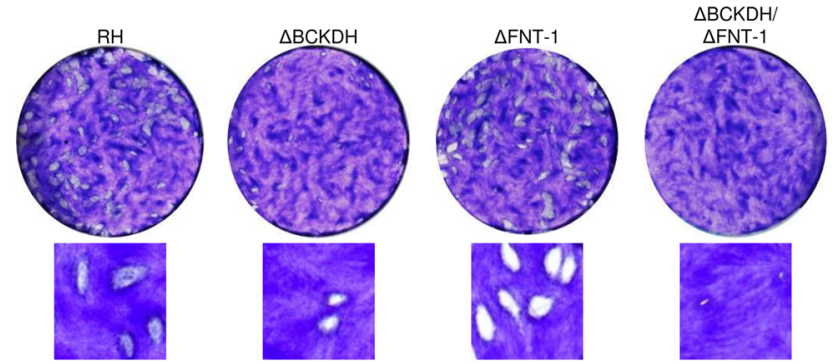

C

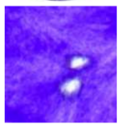

d

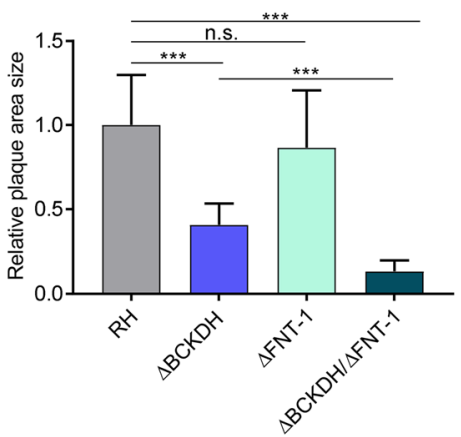

e

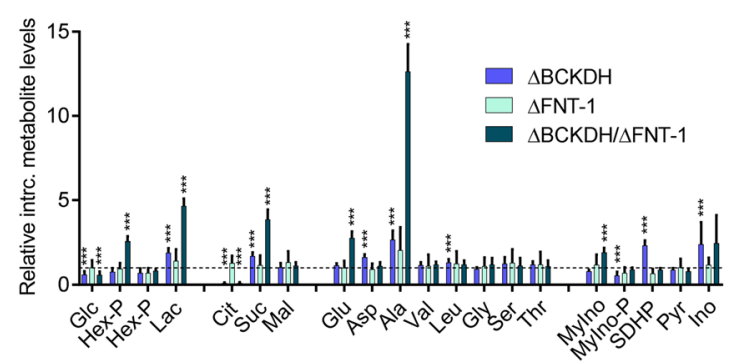

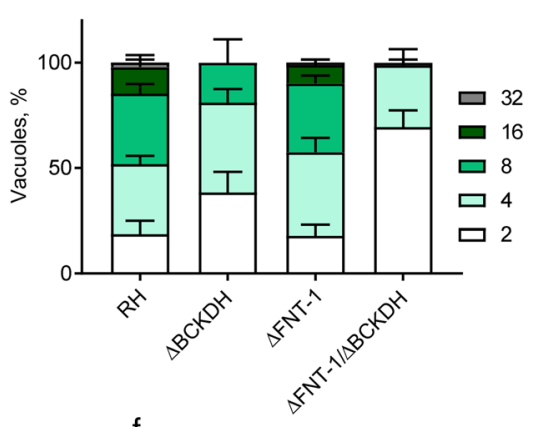

f

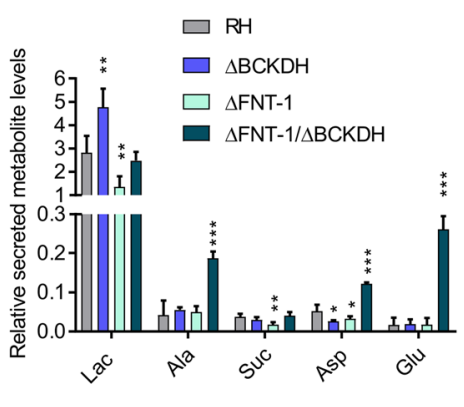

Fig. 8 FNT-1 is dispensable in wild-type T. gondii but becomes highly fitness-conferring in $\triangle B C K D H$ parasites. a Table highlighting the conservation of formate/nitrite transporters (FNTs) across apicomplexans and their fitness score from a recent screen fitness screen of metabolic genes. $\mathbf{b}$ Plaque assay, testing the fitness during the lytic cycle of RH parasites and mutant parasites lacking BCKDH, FNT-1 or both. $\mathbf{c}$ Quantification of plaque area size comparing different strains: $R H, \triangle B C K D H, \Delta F N T-1$ and $\triangle B C K D H / \triangle F N T-1$ double-KO parasites. Error bars represent the standard deviation between 3 independent infections. Per infection, the areas of $>20$ plaques were quantified, and statistically significant differences were determined by a t-test comparing the mutants as indicated (n.S., non-significant; ${ }^{* * *} p<0.001$ ). $\mathbf{d}$ Intracellular growth assay of RH and mutant parasites over $36 \mathrm{~h}$. Error bars represent the standard deviation between 3 independent infections. Per infection, $>100$ vacuoles were counted. e, f Metabolomic analysis of $T$. gondii RH and mutant parasites ( $\triangle B C K D H, \triangle F N T-1$ and $\triangle B C K D H / \triangle F N T-1)$. Levels of intracellular metabolites relative to levels in $\mathrm{RH}$ (dashed line) (e) and of metabolites secreted into the medium by purified parasites normalised to valine, an essential amino acid present in the culture medium at $0.8 \mathrm{mM}(\mathbf{f})$. Error bars in $\mathbf{e}$ and $\mathbf{f}$ represent the standard deviation between replicates $(n=4)$. Statistically significant differences between each mutant and RH were assessed using a $t$-test and are indicated $\left({ }^{*} p<0.05 ;{ }^{* *} p<\right.$ $\left.0.005 ;{ }^{* * *} p<0.001\right)$. BCKDH, branched-chain a-keto acid dehydrogenase-complex; Glc, glucose; Hex-P, hexose-phosphate; Lac, lactate; Cit, citrate; Suc, succinate; Mal, malate; Glu, glutamate; Asp, aspartate; Ala, alanine; Val, valine; Leu, leucine; Gly, glycine; Ser, serine; Thr, threonine; Mylno, myoinositol; Mylno-P, myo-inositol-phosphate; SDHP, sedoheptulose-7-phosphate; Pyr, pyrimidine; Ino, inosine

$\triangle$ FNT-1 exported lactate at similar levels as RH but secreted markedly higher levels of alanine, aspartate and glutamate (Fig. 8f). Our findings highlight that lactate is exported in cells devoid of $\Delta \mathrm{FNT}-1$, consistent with the presence of other lactate transporters FNT-2 and FNT-3 [51]. However, secretion of lactate is significantly decreased in $\triangle$ FNT-1 compared to $\mathrm{RH}$ cells and in $\triangle \mathrm{BCKDH} / \triangle \mathrm{FNT}-1$ compared to $\triangle \mathrm{BCKDH}$ parasites, 
confirming a prominent role of FNT-1 in efficient lactate secretion. Unable to fuel pyruvate after conversion to acetyl-CoA into the TCA cycle and secreting lactate inefficiently, these cells accumulate toxic intracellular levels of lactate and alanine. Our data highlight the metabolic flexibility of $T$. gondii to adapt to obstructions at the transcriptional, translational and post-translational levels.

\section{Discussion}

Enzymes generating acetyl-CoA, as well as enzymes involved in modulating histone acetylation (histone acetylases and deacetylases), have been proposed as drug targets in apicomplexan parasites $[17,32,56]$ and play a crucial role in $T$. gondii development [35]. However, we know little about how acetylation regulates gene expression and enzyme activities and how acetyl-CoA contributes to the metabolism in these pathogens. We reveal here that cytosolic acetyl-CoA affects the levels of various transcripts and proteins. Additionally, we demonstrate that it is required for the elongation of FAs. T. gondii synthesises monounsaturated long and very longchain FAs (FA C20:1, C26:1, C28:1), which are of low abundance or absent in the host cell, making the FA elongation pathway in T. gondii essential [25]. While a previous study reported that depletion of ACS impacts on the FA elongation pathway in T. gondii, the crucial monounsaturated very long-chain FAs (FA C26:1, C28:1) were not detected in the study by Dubois and colleagues, and the phenotype was very modest given the compensatory effect of ACL [57]. FAs generated by FASII as well as their derivatives generated through the elongation pathway have a fundamental role for the completion of $T$. gondii cytokinesis and pellicle formation between the emerging daughter cells [49]. Hence, inhibition of the FA elongation pathway may partially contribute to the described amorphic phenotype of cells devoid of ACS and ACL.

In contrast to the essential pool of nucleo-cytosolic acetyl-CoA, loss of mitochondrial acetyl-CoA can be tolerated by $T$. gondii at a modest fitness cost [13]. We reveal here that lack of BCKDH causes relatively few specific changes in the acetylome, transcriptome and proteome, some of which are crucial to enable these parasites to tolerate obstruction of the link between glycolysis and the TCA cycle. MacRae et al. have proposed that the portion of pyruvate entering the TCA cycle in T. gondii is about $20 \%$ under regular culture conditions [46], while the majority is secreted as lactate, through FNT-1 or FNT-2, the two lactate transporters expressed in tachyzoites [51]. In contrast, Plasmodium parasites, which rely on glycolysis during their intraerythrocytic development, express a single FNT, depletion/inhibition of which leads to parasite death, due to the toxic accumulation of lactate $[52,53]$. In $T$. gondii, a genome-wide fitness screen using CRISPR-Cas 9 reported low positive fitness indices for FNT-1 (1.10) and FNT-2 (1.31), suggesting that both genes are individually dispensable [11]. Indeed, we were able to deplete FNT-1 in tachyzoites without causing any fitness defect. Importantly, these parasites continued to secrete lactate, highlighting that FNT-2 secretes lactate sufficiently under normal culture conditions, compensating for the loss of FNT-1. However, depletion of FNT-1 in cells devoid of $\mathrm{BCKDH}$ resulted in a severe additional fitness defect, and double-KO parasites accumulated very high levels of intracellular lactate. We propose that the identified hypoacetylation of LDH1, as well as the overexpression of FNT-1 in cells lacking BCKDH, is part of a coping mechanism which enables increased reliance on glycolysis in the absence of a functional TCA cycle. These findings provide insights into how parasites adapt their metabolism in response to genetic or pharmacological obstructions.

\section{Conclusions}

This study combines molecular tools and multi-omics to provide a uniquely integrative and global picture of the diverse roles of acetyl-CoA in Toxoplasma gondii physiology. We demonstrate that loss of nucleo-cytosolic acetyl-CoA results in hypo-acetylation of histones and non-histone proteins causing broad changes in gene expression. Further, we show that the absence of cytosolic acetyl-CoA results in a halt in FA elongation, disabling the synthesis of parasite-specific long-chain monounsaturated FAs which cannot be salvaged from the host.

In contrast to the cytosolic acetyl-CoA pool, loss of mitochondrial acetyl-CoA can be tolerated due to an altered central carbon metabolism [13]. How protozoan parasites remodel their metabolism to adapt to varying environments or to obstructions through genetic alterations or drug treatments is poorly understood. We demonstrate here that loss of mitochondrial acetyl-CoA results in the hypo-acetylation of mitochondrial and other proteins as well as in altered gene expression. We provide evidence that these changes at the transcriptional, translational and post-translational levels serve to adapt the metabolism and cope with the lack of mitochondrial acetyl-CoA. These findings provide unprecedented insights into the plasticity and regulation of the metabolism of $T$. gondii.

\section{Material and methods}

\section{Genetic and cell biology approaches T. gondii culture}

All T. gondii strains are derived from RH in which KU80 has been deleted [58]. Parasites were grown in confluent HFFs and maintained in Dulbecco's modified Eagle medium (DMEM, Life Technology, Invitrogen) supplemented with $5 \%$ foetal calf serum, $2 \mathrm{mM}$ L-glutamine, $25 \mu \mathrm{g} / \mathrm{ml}$ gentamicin and where indicated with $0.5 \mu \mathrm{M}$ Shld-1 [23] in a humidified incubator at $37^{\circ} \mathrm{C}$ and $5 \%$ 
$\mathrm{CO}_{2}$. Specific media for stable isotope labelling experiments are described below.

\section{Cloning of DNA constructs}

Amplifications of DNA fragments for cloning were performed with either the LA Taq (TaKaRa) or the Q5 (New England Biolabs) polymerases, and the primers used for each reaction are listed in Additional file 14: Table S8. Correct integration of the different constructs into the genome of the various strains was determined by genomic PCR using the GoTaq Green Master Mix (Promega) and the primers listed in Additional file 14: Table S8.

\section{Preparation of T. gondii genomic DNA}

Genomic DNA was extracted from extracellular tachyzoites using the Wizard SV genomic DNA purification system (Promega).

\section{Inducible knock-down of ACS}

To direct the insertion of this PCR product, a specific guide RNA (gRNA) vector targeting the ATG start codon of acs (TGME49_266640) was generated using the Q5 site-directed mutagenesis kit (New England Biolabs) and the vector pSAG1::CAS9-GFP-U6::sgUPRT as a template [59]. The UPRT-targeting gRNA was replaced by an acsspecific gRNA using the primer pair 1-2 listed in Additional file 14: Table S8. A PCR fragment was amplified of the DD, fused to a myc-tag (DDmyc) with $5^{\prime}$ and 3' homology sequences to the start ATG of ACS using primers P7/P9 as shown in Additional file 1: Figure S1a,c. (KOD polymerase, Novagen) and pTub8DDmycROM4 as template [60]. Ten micrograms of the gRNA plasmid, together with the precipitated KOD PCR product, was transfected, and parasites were grown in the presence of Shld-1. Parasites expressing the Cas9-GFP were sorted by fluorescence-activated cell sorting (FACS) and cloned into 96-well plates using a cell sorter (MoFlo Astrios, Beckman Coulter). Clones were analysed by IFA to confirm the expression of the DDmycACS fusion protein and grown in the presence or absence of Shld-1 to evaluate the regulation of the protein.

\section{Knock-in construct for epitope tagging at the endogenous locus of pepck-1}

A genomic DNA fragment of the C-terminal part of pepck1 (TGME49_289650) was amplified by PCR using the primer pair 3-4 listed in Additional file 14: Table S8, digested with the restriction enzymes $\mathrm{KpnI} / \mathrm{Sbfl}$ and cloned into the pTUB8MIC13-3Ty-HX [61], using the KpnI and Nsil sites. Prior to transfection, the plasmid was linearised in the middle of the cloned genomic DNA fragment using the NcoI site. For knock-in insertion of these vectors into the $\mathrm{RH}$ and $\triangle \mathrm{BCKDH}$ strains, the hxgprt cassette was substituted with a dhfr-ts cassette using the two SacII sites.

\section{Generation of PEPCK-1 second copy-expressing plasmids and acetylation mimetics}

pTub8-PEPCK-1-L-3Ty and pTub8-PEPCK-1-S-3Ty plasmids expressing a second copy of both long and short isoforms of PEPCK-1, respectively, were generated by amplifying the cDNA of pepck-1 using the primers 9-4 (long) or primers 4-10 listed in Additional file 14: Table S8, digested with the restriction enzymes EcoRI and SbfI and cloned into the KpnI and Nsil sites of pTub8APHN21-3Ty-HXGPRT [62]. To complement $\triangle$ PEPCK-1 with either a wild-type copy of PEPCK-1, acetylation mimetics or de-acetylation mimetics of PEPCK-1, 5'UPRTDHFR-pTub8-PEPCK-1-4myc-3'UPRT was first generated. 5'UPRT-DHFR-pTub8-PEPCK-1-4myc-3'UPRT was generated by amplifying the cDNA of pepck-1 using the primers 4-10 listed in Additional file 14: Table S8, digested with the restriction enzymes EcoRI and SbfI and cloned into the of the pUPRT-pTub8-4myc-3'UPRT plasmid. A dhfr-ts cassette was amplified by PCR using primers 1112, digested by ApaI, sub-cloned into pUPRT-pTub8PEPCK-1-4myc-3'UPRT and predigested by ApaI. Lysines K223, K231 and K591 were mutated to either arginine (de-acetylation mimetic) or glutamine (acetylation mimetic) using the Q5 site-directed mutagenesis kit (New England Biolabs), primer pairs listed in Additional file 14: Table S8 and 5'UPRT-DHFR-pTub8-PEPCK-1-4myc3'UPRT as a template. 5'UPRT-DHFR-pTub8-PEPCK-14myc-K3R-3'UPRT and 5'UPRT-DHFR-pTub8-PEPCK-14myc-K3Q-3'UPRT correspond to second copy expressing vectors where PEPCK-1 lysines K223, K231 and K591 were all sequentially mutated to either arginine or glutamine respectively. Prior to transfection, all PEPCK-1 complementation plasmids were linearised by digestion with NotI-HF and AvrII. Linearised plasmids were cotransfected with $5 \mu \mathrm{g}$ pSAG1::CAS9-GFP-U6::sgUPRT [59]. Transgenic parasites were selected with pyrimethamine and cloned in 96-well plates. Expression of PEPCK1 was validated by IFA.

\section{KO strains}

The KO of ACL (TGME49_223840) and BCKDH-E1a (TGME49_239490) were previously described [13, 14]. To generate the KO of PEPCK-1 (TGME49_289650), a plasmid (pTub-CAT-PEPCK-1-ko) was generated and around $1.5 \mathrm{~kb}$ of the $5^{\prime}$ and $3^{\prime}$ flanking regions of PEPCK-1 were amplified using primer pairs 5-6 and 7-8, respectively. The 5 ' flanking region was then cloned between KpnI and HindIII restriction sites of the pTub5-CAT and the 3' flanking region between the BamHI and NotI sites. The plasmid was cut with KpnI and NotI prior to transfection. The KO of FNT-1 (TGME49_209800) was generated as follows: 2gRNA plasmid for the FNT-1 KO was generated as described previously [16] with primers as described in Additional file 14: Table S8. Forward and reverse primers 
with homology to either the DHFR-TS (p2854-DHFR) selection cassette and to the $5^{\prime}$ and $3^{\prime}$ coding sequence of the gene were generated (KOD polymerase, Novagen). Primers as listed in Additional file 14: Table S8, $10 \mu \mathrm{g}$ of the 2gRNA plasmid, together with the precipitated KOD PCR product, was transfected. Primers to check for successful integration were used on extracted genomic DNA as shown in Additional file 14: Table S8.

\section{Parasite transfection and selection of stable transformants}

Parasite transfections were performed by electroporation as previously described [63]. Either mycophenolic acid (MPA, $25 \mathrm{mg} / \mathrm{ml}$ ) and xanthine $(50 \mathrm{mg} / \mathrm{ml})$, pyrimethamine $(1 \mu \mathrm{g} / \mathrm{ml})$ or chloramphenicol $(20 \mu \mathrm{g} / \mathrm{ml})$ were used to select the resistant parasites carrying the HXGPRT, DHFR-TS or CAT cassette, respectively.

\section{Antibodies}

The antibodies used in this study were previously described as follows: the polyclonal rabbit antibodies used in this study include anti- $(\alpha)$-GAP45 [64] and $\alpha$-TgPRF [65]. Monoclonal mouse antibodies include $\alpha$-TgActin [66], $\alpha$-myc (9E10), $\alpha$-Ty (BB2) [67], $\alpha$-SAG1 (generous gift from Dr. J-F Dubremetz), 5F4 ( $\alpha$-F1B- ATPase, kindly provided by PJ Bradley) [68], $\alpha$-Atrx1 [69] and $\alpha$ acetyl-lysine (Cell Signaling Technology). For western blot analyses, secondary horseradish peroxidase (HRP)conjugated goat $\alpha$-rabbit or $\alpha$-mouse antibodies (Sigma) were used. For IFAs, the secondary antibodies Alexa Fluor 488- and Alexa Fluor 594-conjugated goat $\alpha$ mouse or goat $\alpha$-rabbit antibodies (Life Technologies) were used.

\section{IFAs and confocal microscopy}

$\mathrm{i} \triangle \mathrm{ACS}$ or $\triangle \mathrm{ACL} / \mathrm{i} \triangle \mathrm{ACS}$ parasites were grown in HFF cells seeded on coverslips for $24 \mathrm{~h}$ in the presence or absence of Shld-1. Similarly, stable transgenic parasite lines were grown in HFFs seeded on coverslips. Cells were fixed with $4 \%$ paraformaldehyde $/ 0.05 \%$ glutaraldehyde (PFA/GA) in phosphate-buffered saline (PBS) for $10 \mathrm{~min}$ at room temperature. Fixed cells were then processed as previously described [70]. Confocal images were generated with a Zeiss LSM700 laser scanning confocal microscope using an apochromat $\times 63 / 1.4$ oil objective. Image stacks were processed with ImageJ and projected using the maximum projection tool.

\section{Western blot analyses}

$\mathrm{i} \triangle \mathrm{ACS}$ and $\triangle \mathrm{ACL} / \mathrm{i} \triangle \mathrm{ACS}$ intracellular parasites were incubated in the presence or absence of Shld-1 for varying durations as indicated. Freshly egressed parasites were lysed in radioimmunoprecipitation assay (RIPA) buffer (150 mM sodium chloride $(\mathrm{NaCl}), 1 \%$ Triton $\mathrm{X}-100,0.5 \%$ deoxycholate, $0.1 \%$ SDS and $50 \mathrm{mM}$ Tris(hydroxymethyl)- aminomethan (TRIS) pH 7.5) for $10 \mathrm{~min}$ on ice and mixed with sodium dodecyl sulfate (SDS)-polyacrylamide gel electrophoresis (PAGE) loading buffer under reducing conditions and subjected to sonication. Protein lysates were separated by SDS-PAGE and transferred onto nitrocellulose membranes before blocking in 5\% non-fat milk in PBS- $0.05 \%$ Tween- 20 or TRIS-buffered saline with Tween-20 (TBST), followed by incubation of the primary antibody diluted in 5\% milk-TBST.

\section{Transmission electron microscopy}

$\mathrm{RH}$ and $\triangle \mathrm{ACL} / \mathrm{i} \triangle \mathrm{ACS}$ parasites were inoculated on confluent HFFs and allowed to grow for $24 \mathrm{~h}$ in the presence or absence of Shld-1. Infected host cells were washed with $0.1 \mathrm{M}$ phosphate buffer $\mathrm{pH} 7.4$, then fixed with $2.5 \% \mathrm{GA}$ in $0.1 \mathrm{M}$ phosphate buffer $\mathrm{pH} 7.4$, scraped and pelleted. Samples were further processed and examined using a Technai 20 electron microscope (FEI Company) as described before [68].

\section{Transcriptomic analysis Extraction of RNA}

$\mathrm{RH}$ and $\triangle \mathrm{ACL} / \mathrm{i} \triangle \mathrm{ACS}$ (following $16 \mathrm{~h}$ growth in the absence of Shld-1) or $\triangle B C K D H$ parasites were analysed. Biological triplicates of infected HFFs were collected following a rinse with cold PBS and pelleted by centrifugation at $(1000 g, 10 \mathrm{~min})$. Total RNA from the samples was isolated using a hybrid RNA extraction protocol with TRIzol (Life Technologies) and QIAGEN RNeasy Mini Kit. First, sample pellets were lysed in TRIzol followed by the separation of aqueous/organic fractions with the addition of chloroform. RNA from the aqueous phase was precipitated with $70 \%$ ethanol, loaded onto RNeasy columns and further processed according to the manufacturer's instructions.

\section{RNA sequencing and data analysis}

Isolated RNA was subjected to 100-bp single read sequencing on an Illumina HiSeq 2500 at the iGE3 genomics platform of the University of Geneva (http://www. ige3.unige.ch/genomics-platform.php) (Genomics platform, Institute of Genetics and Genomics iGE3, University of Geneva). Two sets of samples $(2 \times 3$ replicates $)$ were loaded per sequencing lanes of the flow cell. Adapter sequences from the raw reads were trimmed using FASTX-Toolkit (http://hannonlab.cshl.edu/fastx toolkit/) (phred $<20)$. Following quality control, resulting reads were aligned to the $T$. gondii reference genome (ToxoDB-12) with TopHat/Bowtie2 aligner, and HTSeqcount was used to generate the read counts of the genes [71-74]. edgeR, a Bioconductor package in R (http:// www.R-project.org), was implemented to do differential expression analysis. All computations were performed 
on the Baobab cluster at the University of Geneva. The dataset is available in a data repository [48].

\section{Proteomic analyses \\ Sample preparation}

$\mathrm{RH}$ and $\triangle \mathrm{ACL} / \mathrm{i} \triangle \mathrm{ACS}$ (following $16 \mathrm{~h}$ growth in the absence of Shld-1) or $\triangle \mathrm{BCKDH}$ parasites were analysed. Freshly egressed parasites were lysed in $8 \mathrm{M}$ urea and 50 $\mathrm{mM}$ (4-(2-hydroxyethyl)-1-piperazineethanesulfonic acid) (HEPES). Extracted proteins were reduced using $20 \mathrm{mM}$ of dithiothreitol for $1 \mathrm{~h}$ at $37^{\circ} \mathrm{C}$ before alkylation with 55 $\mathrm{mM}$ of iodoacetamide for $45 \mathrm{~min}$ at room temperature in the dark. The samples were then diluted using ammonium bicarbonate to obtain a urea concentration of $4 \mathrm{M}$. Proteins were digested with LysC (Promega) at a ratio of 1 : 200 during $4 \mathrm{~h}$ at $37^{\circ} \mathrm{C}$. The samples were diluted again using ammonium bicarbonate to obtain a urea concentration of $1 \mathrm{M}$. Proteins were then digested with Trypsin (Promega) at a ratio of 1:200 overnight at $37^{\circ} \mathrm{C}$. Resulting peptides were purified by $\mathrm{C} 18$ reverse phase chromatography (Sep-Pak C18, Waters) before drying down. For the comparison of the total proteomes of the $\triangle \mathrm{ACL} / \mathrm{i} \triangle \mathrm{ACS}$ mutant strain and its parental RH strain, the samples were further fractionated by tip-based strong cation exchange (3M Empore). Briefly, peptides were dissolved in 5\% acetonitrile and $1 \%$ trifluoracetic acid (TFA) and eluted in 4 fractions (F1: $100 \mathrm{mM}$ ammonium acetate, $20 \%$ acetonitrile, $0.5 \%$ formic acid; F2: $175 \mathrm{mM}$ ammonium acetate, $20 \%$ acetonitrile, $0.5 \%$ formic acid; F3: $375 \mathrm{mM}$ ammonium acetate, $20 \%$ acetonitrile, $0.5 \%$ formic acid; F4: $80 \%$ acetonitrile, 5\% ammonium hydroxide) before desalting using C18 reverse phase chromatography (Ultra-Micro SpinColumns, Harvard Apparatus). Technical triplicates were performed.

\section{Enrichment of acetylated peptides}

Dried peptides were dissolved in IP buffer $(100 \mathrm{mM}$ $\mathrm{NaCl}, 1 \mathrm{mM}$ ethylenediaminetetraacetate (EDTA), 20 $\mathrm{mM}$ TRIS-HCl, $0.5 \% \mathrm{NP}-40, \mathrm{pH}$ 8). Acetylated peptides were mixed with $\alpha$-acetyl-lysine antibody immobilised on agarose beads (ImmuneChem-ICP0388) and incubated overnight at $4{ }^{\circ} \mathrm{C}$. Following incubation, the beads were washed twice with IP buffer, once with washing buffer (IP buffer without NP-40) and twice with ice cold ultra-pure water. Elution was performed using $0.1 \%$ TFA. Enriched peptides were then dried down. Three technical replicates were prepared from each sample.

\section{NanoLC-MS/MS analyses}

NanoLC-MS/MS analyses were performed using an Ultimate 3000 RSLCnano coupled to a Q-Exactive Plus (Thermo Scientific). Peptides were sampled on a $300 \mu \mathrm{m} \times 5 \mathrm{~mm}$ PepMap C18 precolumn and separated on a PepMap $75 \mu \mathrm{m} \times 250 \mathrm{~mm}$ C18 column $(2 \mu \mathrm{m}$,
Thermo Scientific). The nanoLC methods consisted of 120 or $240 \mathrm{~min}$ gradients at a flow rate of $300 \mathrm{nl} / \mathrm{min}$ for analysis of respectively enriched acetylated peptides, strong cation exchange fractions and unfractionated total proteome. Spray voltage was set at $1.6 \mathrm{kV}$, and heated capillary was adjusted to $250-270{ }^{\circ} \mathrm{C}$.

For acetylome analyses, survey full-scan MS spectra $(m / z 400-1600)$ were acquired with a resolution of 70 , 000, with AGC target set to $10^{6}$ ions (maximum filling time $250 \mathrm{~ms}$ ) and with lock mass option activated. The 10 most intense ions were fragmented by higher-energy collisional dissociation (nce $=30$ ) with a resolution of 17 , 500 , with AGC target set to $10^{6}$ ions (maximum filling time $250 \mathrm{~ms}$ ). Analytical triplicates were acquired for the comparison of acetylomes between $\triangle \mathrm{BCKDH}$ mutant strain and its parental RH strain.

For total proteome analyses, survey full-scan MS spectra $(m / z 400-1600)$ were acquired with a resolution of 70,000 , with AGC target set to $10^{6}$ ions (maximum filling time $200 \mathrm{~ms}$ ) and with lock mass option activated. The 10 most intense ions were fragmented by higherenergy collisional dissociation $(\mathrm{nce}=30)$ with a resolution of 17,500 , with AGC target set to $10^{5}$ ions (maximum filling time $50 \mathrm{~ms}$ ). MS and tandem MS data were acquired using the Xcalibur software (Thermo Scientific). Analytical quadruplicates were acquired for comparison of total proteomes of $\triangle \mathrm{BCKDH}$ mutant strain and its parental RH strain.

Raw and processed MS data have been deposited in a data repository $[28,75]$.

\section{Peptide, protein and acetylated site identifications}

RAW files were processed using MaxQuant [76] version 1.5.8.3. Spectra were searched against the $T$. gondii database (ME49 taxonomy, version 30 downloaded from ToxoDB [77], the Swiss-Prot database (Homo sapiens taxonomy, 2017-08-24 version), the frequently observed contaminants database embedded in MaxQuant and the corresponding reverse databases. Trypsin was chosen as the enzyme, and two or three missed cleavages were allowed for respectively total proteome and acetylome analyses. Precursor and fragment mass error tolerances were set at their default values. Peptide modifications allowed during the search were carbamidomethyl (C, fixed), acetyl (protein $\mathrm{N}$-term, variable), oxidation ( $\mathrm{M}$, variable) for total proteome analyses, and acetyl (K, variable) was added for acetylome analyses. Minimum number of peptides, razor + unique peptides and unique peptides were all set to 1 . Maximum false discovery rates were set to 0.01 at PSM and site levels for acetylome analyses and at 0.01 at PSM, protein and site levels for total proteome analyses. The match between runs option was selected. For total proteome analyses, label-free protein quantitation (LFQ) was performed with a minimum 
ratio count of 2 . For acetylome analyses, only acetylated sites with a probability above 0.9 were considered.

\section{Statistical analyses}

Statistical analyses were performed using ProStaR [78]. Peptides and proteins identified in the reverse and contaminant databases or matching to human sequences were discarded. For the comparison of acetylomes between $\triangle B C K D H$ mutant strain and its parental $\mathrm{RH}$ strain, intensities of the acetylated sites were deduced through averaging the values extracted from the analytical triplicates. For differential analysis of acetylomes, acetylated sites were split into several lanes when they were identified on peptides bearing various numbers of acetylated sites to allow the quantification of the different peptidoforms. Only proteins and acetylated sites detected in at least 3 replicates of one condition were conserved. After $\log 2$ transformation, protein LFQ intensities and acetylated site intensities were median normalised before missing value imputation (replacing missing values by the first percentile value of each column). Statistical testing was conducted using limma. Differentially abundant proteins and acetylated sites were sorted out using the following cut-offs: $\log 2$ (fold change) $\geq 1$ or $\leq-1$ and $p$ value $<0.01$, allowing to reach an FDR inferior to $5 \%$ according to the BenjaminiHochberg estimator.

\section{GO enrichment analyses}

GO enrichment analyses of transcripts and proteins was carried out using the tool for enrichment in biological processes on ToxoDB (https://toxodb.org/) against the total $T$. gondii database with a $p$ value cut-off of 0.05 (limited to GO slim terms function). GO enrichment analysis of the acetylome was performed using the $\mathrm{R}$ package, topGO (version 2.38.1) [29]. Enrichment factors were calculated as the ratio of the significant genes assigned to the GO to the expected number of assigned genes defined by topGO. Enrichments in biological processes were determined in comparison with either the total $T$. gondii genome/proteome ( $p$ value cut-off, 0.001 ) or against the subset of identified acetylated proteins $(p$ value cut-off, 0.05).

\section{Metabolomic analyses \\ Metabolomics sample preparation}

Triplicates of RH, i $\Delta \mathrm{ACS}$ and $\triangle \mathrm{ACL} / \mathrm{i} \Delta \mathrm{ACS}$ intracellular parasites were incubated in the absence of Shld-1 for 16 h. Freshly egressing parasites were extracted through repeated syringe lysis $(3 \times, 28 \mathrm{G})$, purified from host cell material through filtration ( $3 \mu \mathrm{m}$ pore size, Millipore/ Merck) and pelleted by centrifugation $(2800 \mathrm{~g}, 20 \mathrm{~min}$, $\left.4{ }^{\circ} \mathrm{C}\right)$. Pellets were washed with ice-cold PBS $(3 \times)$, and metabolites were extracted as outlined below.

\section{Untargeted LC-MS analysis}

Metabolites were extracted in $80 \%$ acetonitrile and 20\% ultra-pure water through vigorous vortexing. An aliquot $(10 \mu \mathrm{l})$ of each sample was combined to generate a pooled biological quality control (PBQC) sample, which was used to monitor downstream sample stability and analytical reproducibility. Metabolomics analysis was performed by LC-MS, using hydrophilic interaction liquid chromatography (ZIC-pHILIC, Merck) and highresolution MS (Q-Exactive Orbitrap, Thermo Fisher) as previously described [79]. Sample injections within the experiment were randomised to avoid any impact of systematic instrument drift on metabolite signals. Retention times for 250 authentic standards were checked manually to aid metabolite identification.

Identification and quantification of metabolites were performed using the IDEOM workflow [80]. Over 850 metabolites were putatively identified based on accurate mass and standard retention time (where available) (confidence score 1 in Additional file 2: Table S1), or predicted retention time (confidence score 2 in Additional file 2: Table S1) [81]. Metabolite abundance was determined by LCMS peak height and was normalised to the average for wild-type (RH) samples. Statistical analyses utilised Welch's $t$-test $(\alpha=0.05)$, and pathway analysis used KEGG annotations (Microsoft Excel). Stable isotope labelling was analysed using mzMatch-ISO [82] to extract all isotopologues of all putatively identified metabolites based on retention time and accurate mass. The complete dataset is available in a data repository [24].

\section{FA profiling by GC-MS}

$\mathrm{RH}$ and $\triangle \mathrm{ACL} / \mathrm{i} \triangle \mathrm{ACS}$ intracellular parasites were incubated in the absence of Shld- 1 for $16 \mathrm{~h}$ and simultaneously labelled with either $10 \mathrm{mM} \mathrm{U-}{ }^{13} \mathrm{C}$-glucose or 2 $\mathrm{mM} \mathrm{U}{ }^{13} \mathrm{C}$-acetate (Cambridge Isotope Laboratories). Per sample, $10^{8}$ parasites were isolated and purified as described above. FAs were extracted, derivatised and analysed as described previously [16]. Fatty acid methyl esters (FAMEs) were identified based on retention times and the library integrated in Xcalibur (Thermo Fisher Scientific). The abundance was determined based on the peak intensity relative to the cholesterol signal intensity in each sample. Abundance of the C14:0 and C26:1FAME mass isotopologues $(\mathrm{m} / z$ 242-256; $\mathrm{m} / z$ 376-390) was determined using Xcalibur (Thermo Fisher Scientific) and OpenChrom software. The extent of ${ }^{13} \mathrm{C}$-labelling was determined using the Excel (Microsoft) software following correction for the occurrence of natural isotopes as described by Zamboni et al. [83]. Abundance data represent the average of 6 biological replicates, and labelling data represents the average of 3 biological replicates. Statistical significance of differences in labelling and abundance were assessed by $t$-test. 
Standard deviation and $p$ values are indicated in the figure.

\section{Polar metabolite profiling in PEPCK-1 acetylation mimetics} Intracellular parasites were cultured in the presence of equimolar $(8 \mathrm{mM})$ levels of natural-abundance glucose and $\mathrm{U}^{13}{ }^{13} \mathrm{C}$-glutamine (Cambridge Isotope Laboratories) for $24 \mathrm{~h}$. Freshly egressing parasites $\left(10^{8}\right.$ cells $)$ were purified and harvested and metabolites extracted as described above. Polar metabolite extracts were prepared and analysed as described previously [16]. Aspartate and glucose-6-phosphate were identified based on their retention time and ion spectrum of authentic standards. The signal for mass isotopologues of major ions of aspartate $(m / z 232-235)$ and glucose-6-P $(m / z 471-475)$ was determined using Xcalibur (Thermo Fisher Scientific) and OpenChrom software. The extent of ${ }^{13} \mathrm{C}$-labelling in these ions was determined using the Excel (Microsoft) software following correction for the occurrence of natural isotopes as described by Zamboni et al. [83]. Displayed data represent the average of 3 biological replicates. The standard deviation is indicated.

\section{Profiling of intracellular and secreted metabolites in $\triangle B C K D H$ and $\triangle F N T-1$ cells}

Freshly egressing tachyzoites $\left(10^{8}\right.$ parasites $)$ of each strain were harvested and purified as described above and incubated $\left(3 \mathrm{~h}, 37^{\circ} \mathrm{C}\right)$ in $1.5 \mathrm{ml}$ of glucose-free DMEM (Gibco) supplemented with $12 \mathrm{mM} \mathrm{U}{ }^{13} \mathrm{C}$-glucose (Cambridge Isotope Laboratories). Subsequently, parasites were rapidly chilled and pelleted by centrifugation $\left(6000 \mathrm{~g}, 10 \mathrm{~min}, 4^{\circ} \mathrm{C}\right)$. Culture medium was taken from the supernatant and stored at $-80^{\circ} \mathrm{C}$. Cell pellets were washed $3 \times$ with PBS and intracellular metabolites extracted as described previously [16] with the polar phase $(300 \mu \mathrm{l})$ containing $5 \mu \mathrm{M}$ scyllo-inositol (SigmaAldrich/Merck) as an internal standard. Twenty microlitres of cell culture medium and polar metabolite extracts was dried down in MS vial inserts using a centrifugal evaporator. The dried medium samples and cell extracts were derivatised using methoxyamine hydrochloride (Sigma-Aldrich/Merck) and N,O-bis(trimethylsilyl)trifluoroacetamide trimethylchlorosilane (Sigma-Aldrich/ Merck) as described previously [16]. Metabolites were identified based on the retention time and ion spectrum of authentic standards and were quantified by integrating the area under the peak in the total ion chromatogram (TIC) using Xcalibur (Thermo Fisher Scientific) and OpenChrom software and comparing their abundance to that of the internal standard scyllo-inositol (intracellular metabolites) or to the abundance of valine (medium samples). The relative abundance of metabolites was determined using the Excel (Microsoft) software. Displayed data represent the average of 3-5 biological replicates. Standard deviation between replicates is indicated by the error bars, and statistically significant differences were tested by $t$-test and are as indicated in the figure.

\section{Supplementary information}

Supplementary information accompanies this paper at https://doi.org/10. 1186/s12915-020-00791-7.

Additional file $\mathbf{1}$ : Figure $\mathbf{S} \mathbf{1}$. Generation of $\triangle A C L$ and i $\triangle A C S$ parasites. PDF image showing a schematic representations of the strategies used to introduce a DDmyc destabilisation domain at the ATG of the locus of acs generating i $\triangle \mathrm{ACS}(\mathbf{a})$ and to delete $\operatorname{acl}(\triangle \mathrm{ACL})$ using double homologous recombination (b), scheme adapted from [14]. PCRs were performed on genomic DNA extracted from clones and using primers listed in Additional file 14: Table S8, confirming correct integration of the constructs (c). Abbreviations: GOI: gene of interest; FS: flanking sequence; gRNA: guide RNA; ACL: ATP-citrate lyase; ACS: acetyl-CoA synthetase; HXGPRT: hypoxanthine-xanthine-guanine phosphoribosyl transferase; DD: destabilization domain; PCR: polymerase chain reaction; DNA: deoxyribonucleic acid.

Additional file 2 : Table S1. $\triangle \mathrm{ACL} / \mathrm{i} \triangle \mathrm{ACS}$ metabolome. Excel spreadheet summarising the metabolomic data set from the LC-MS analysis. Metabolites which are significantly decreased or increased in $\triangle A C L$ parasites, i $\triangle \mathrm{ACS}$ parasites and $\triangle \mathrm{ACL} / \mathrm{i} \triangle \mathrm{ACS}$ parasites are highlighted. Abbreviations: ACL: ATP-citrate lyase; ACS: acetyl-CoA synthetase; LC-MS: Iiquid chromatography-mass spectrometry.

Additional file $\mathbf{3}$ : Figure S2. Alterations in metabolite abundances and FA labelling in $\triangle \mathrm{ACL} / \mathrm{i} \triangle \mathrm{ACS}$ parasites. PDF image providing an (a) overview of metabolites which were significantly altered in their abundance in $\triangle \mathrm{ACL} / \mathrm{i} \triangle \mathrm{ACS}$ parasites compared to $\mathrm{RH}(\mathrm{RH}$ abundance $=$ 1). Metabolites were grouped according to their class. Error bars indicate the standard deviation between triplicates. All displayed metabolites were found to be significantly de- or increased by t-test $(p<0.05)$. (b) Fractional ${ }^{13} \mathrm{C}$-labelling from $\mathrm{U}-{ }^{13} \mathrm{C}$-glucose $\left(\mathrm{U}-{ }^{13} \mathrm{C}\right.$-GlC) and $\mathrm{U}-{ }^{13} \mathrm{C}$-acetate $\left(\mathrm{U}-{ }^{13} \mathrm{C}-\mathrm{AC}\right)$ in myristate (FA C14:0) and FA C26:1 was measured by GC-MS following labelling of $\mathrm{RH}$ and $\triangle \mathrm{ACL} / \mathrm{i} \triangle \mathrm{ACS}$ parasites for 16 hours during simultaneous ACS depletion. Error bars represent the standard deviation between replicates. Statistical significance was test using a t-test and is as indicated $\left(*-p<0.05 ;{ }^{* * *}-p<0.0001\right)$. Abbreviations: ACL: ATP-citrate lyase; ACS: acetyl-CoA synthetase; FA: fatty acid; GC-MS: gas chromatography-mass spectrometry; Glc: glucose; Ac: acetate.

Additional file 4 : Figure $\mathbf{S 3}$. Stable isotope labelling to elucidate the consequences of the loss of ACL and/or ACS. PDF image showing (a) Fractional ${ }^{13} \mathrm{C}$-labelling from $U-{ }^{13} \mathrm{C}$-glucose in glycolysis and TCA-cycle intermediates comparing $\mathrm{RH}, \triangle \mathrm{ACL} \mathrm{i} \triangle \mathrm{ACS}$ or $\triangle \mathrm{ACL} / \mathrm{i} \triangle \mathrm{ACS}$ parasites. The in ducible knock-down parasites were grown in the absence of Shield-1 for 16 hours prior to analysis. (b) Fractional ${ }^{13} \mathrm{C}$-labelling from $\mathrm{U}-{ }^{13} \mathrm{C}$-acetate in glycolysis and TCA-cycle intermediates. (c-f) Mass isotopologue abundance of selected metabolites following labelling with $\mathrm{U}^{13} \mathrm{C}$-acetate: citrate $(\mathbf{c})$, ceramide $\operatorname{Cer}(36: 1)(\mathbf{d})$, phosphatidylserine $\mathrm{PS}(36: 2)(\mathbf{e})$ and PS(38:2) (f). Legend in c-e as in f. Abbreviations: TCA: tricarboxylic acid; ACL: ATP-citrate lyase; ACS: acetyl-CoA synthetase.

Additional file $\mathbf{5}$ : Figure S4. Acetylome analysis of $\triangle A C L / i A C S$ and $\triangle \mathrm{BCKDH}$ parasites. PDF image of the (a) Western blot analysis representing the total lysine acetylation profile of $\triangle \mathrm{ACL} / \mathrm{i} \triangle \mathrm{ACS}$ parasites. $\triangle \mathrm{ACL} / \mathrm{i} \triangle \mathrm{ACS}$ parasites were cultured in the absence of Shield-1 for 16 hours prior to harvest and analysis. The blot was probed with a-acetyllysine antibody for the total lysate and a-actin as loading control. (b) Western blot analysis representing the total lysine acetylation profile of $\triangle \mathrm{BCKDH}$ parasites. The blot was probed with a-acetyl-lysine antibody for the total lysate and a-actin as loading control. (c) The localisation of all differentially acetylated proteins in $\triangle \mathrm{ACL} / \mathrm{i} \triangle \mathrm{ACS}$ parasites is shown. Subcellular localisation of differentially acetylated proteins and enrichment was determined using the hyperplexed Localisation of Organelle Proteins by Isotopic Tagging (hyperLOPIT) data available under https://proteome. shinyapps.io/toxolopittzex/. (d) The localisation of differentially acetylated 
proteins in $\triangle B C K D H$ parasites was determined as above and is displayed. (e) Proteins identified as differentially acetylated in $\triangle \mathrm{ACL} / \mathrm{I} \triangle \mathrm{ACS}$ parasites were analysed using GO-enrichment R-package topGO to identify enrichment in biological processes using the relatively small subset of acetylated proteins as background. Enrichment was 1.3- (GO:0044267 - cellular protein metabolic process) and 1.9-fold (GO:0051276 - chromosome organization). Statistically significant enrichment was assessed by Fisher's exact test ( $p$-value $<0.05)$. (f) Proteins identified as differentially acetylated in $\triangle \mathrm{BCKDH}$ parasites were analysed using GO-enrichment R-package topGO to identify enrichment in biological processes using the relatively small subset of acetylated proteins as background. Enrichment ranged from 2.3- (GO:0072350 - tricarboxylic acid metabolic process) to 1.6-fold (all other enriched biological processes). Statistically significant enrichment was assessed by Fisher's exact test ( $p$-value $<0.05$ ). Abbreviations: BCKDH: branched-chain a-keto acid dehydrogenase-complex; ACL: ATPcitrate lyase; ACS: acetyl-CoA synthetase; PM: plasma membrane; IMC: inner membrane complex; ER: endoplasmic reticulum.

Additional file $\mathbf{6}$ : Table S2. $\triangle \mathrm{ACL} / \mathrm{i} \triangle \mathrm{ACS}$ acetytlome. Excel spreadheet summarising the acetylome data set from the nanoLC-MS/MS analysis. Sites and proteins which are significantly hypo- or hyper-acetylated in $\triangle \mathrm{ACL} / \mathrm{i} \mathrm{ACS}$ parasites are highlighted. Abbreviations: ACL: ATP-citrate lyase; ACS: acetyl-CoA synthetase; LC-MS/MS: liquid chromatographytandem mass spectrometry.

Additional file 7 : Table S3. $\triangle B C K D H$ acetytlome. Excel spreadheet summarising the acetylome data set from the nanoLC-MS/MS analysis. Sites and proteins which are significantly hypo- or hyper-acetylated in $\triangle B C K D H$ parasites are highlighted. Abbreviations: $B C K D H$ : branched-chain a-keto acid dehydrogenase-complex; LC-MS/MS: liquid chromatographytandem mass spectrometry.

Additional file 8 : Figure S5. Generation and analysis of PEPCK-1-3Ty knock-in, $\triangle$ PEPCK-1 strains and PEPCK-1 acetylation mimetics in T. gondii. PDF file showing the (a) schematic of the putative mechanism of gluconeogenesis-activation through PEPCK-1 de-acetylation. Gluconeogenesis is inactive in wild-type parasites $(\mathrm{RH})$ under glucose replete conditions (left panel), during which PEPCK is acetylated in position K223. Inactivity of the gluconeogenesis pathway if graphically represented by the faint dashed arrow and PEPCK-1 acetylation is graphically represented by an orange circle. We identified PEPCK-1 to be hypo-acetylated in $\triangle B C K D H$ parasites (right panel) and a previous study highlighted the constitutive activation of gluconeogenesis in parasites lacking BCKDH [13]. Activation of gluconeogenesis is represented by the thick arrow. These findings prompted us to hypothesize that PEPCK-1 acetylation in lysine 223 may be responsible for the activation of the pathway and to assess the role of gluconeogenesis in parasites lacking BCKDH. (b) Schematic representation of the pepck-1 locus. Two alternative translational starts are represented by inverted triangles (blue) while the translational stop is represented by a red triangle. (c) Secondary expression of the long isoform of PEPCK-1 (PEPCK-1-3Ty-L, mitochondrial) and the short PEPCK-1 (PEPCK-1-3Ty-S, cytosolic). Expression of the second PEPCK-1 copy was detected using a-Ty (green) while a-gliding associated protein 45 (aGAP45, red) was used as a pellicle marker. (d) Graphical representation of the knock-in strategy used to introduce a 3Ty tag at the C-terminus of the endogenous pepck-1 locus in RH parasites. (e) PCRs performed on genomic DNA extracted from clones showing correct integration of the construct. (f) Schematic representation of the double homologous recombination strategy used to $\mathrm{KO}$ the endogenous pepck-1 locus in $\mathrm{RH}$ and $\triangle B C K D H$ parasites. (g) PCRs performed on genomic DNA using primers listed in Additional file 14: Table S8 show correct integration of the construct. (h) Expression of the acetylation mimetics of PEPCK-1 was tested by IFA using an a-Ty antibody. (i) pepck-1 was replaced in $\triangle B C K D H$ parasites by a cat resistance cassette as shown in panel f. PCRs were performed on genomic DNA extracted from clones showing correct integration of the construct. (j) Plaque assays of $\mathrm{RH}, \triangle \mathrm{BCKDH}, \triangle \mathrm{PEPCK}-1$ or double $\mathrm{KO} \triangle \mathrm{BCKDH} / \triangle \mathrm{PEPCK}-1$ parasites. $(\mathrm{k}, \mathrm{l})$ Intracellular growth assays at 24 hours post infection of RH, $\triangle B C K D H, \triangle P E P C K-1$ and $\triangle B C K D H$ / $\triangle P E P C K-1$ parasites in complete medium ( $\mathrm{k}$ ) or in medium lacking glucose (I). Error bars represent the standard deviation between 3 independent infections. Per infection $>100$ vacuoles were counted. Throughout the figure PEPCK refers to PEPCK-1, the active enzyme in tachyzoites [42].
Abbreviations: GNG: gluconeogenesis; PEP: phosphoenolpyruvate; Gln: glutamine; Cit: citrate; OAA: oxaloacetate; PM: plasma membrane; PEPCK: phosphoenolpyruvate carboxykinase-1; BCKDH: branched-chain a-keto acid dehydrogenase-complex; DHFR-TS: dihydrofolate reductasethymidylate synthase; CAT: chloramphenicol acetyltransferase; FS: flanking sequence; PCR: polymerase chain reaction; DNA: deoxyribonucleic acid.

Additional file 9 : Table S4. $\triangle \mathrm{ACL} / \mathrm{i} \triangle \mathrm{ACS}$ transcriptome. Excel spreadheet summarising the transcriptome data set from the RNA-seq analysis. Transcripts which are significantly up- or downregulated in $\triangle A C L / i \triangle A C S$ parasites are highlighted. Abbreviations: ACL: ATP-citrate lyase; ACS: acetyl-CoA synthetase.

Additional file 10 : Table S5. $\triangle \mathrm{ACL} / \mathrm{i} \triangle \mathrm{ACS}$ proteome. Excel spreadheet summarising the proteome data set from the nano-LC-MS/MS analysis. Proteins which are significantly up- or downregulated in $\triangle A C L / i \triangle A C S$ parasites are highlighted. Abbreviations: ACL: ATP-citrate lyase; ACS: acetyl-CoA synthetase; LC-MS/MS: liquid chromatography-tandem mass spectrometry.

Additional file 11 : Table S6. $\triangle B C K D H$ transcriptome. Excel spreadheet summarising the transcriptome data set from the RNA-seq analysis. Transcripts which are significantly up- or downregulated in $\triangle B C K D H$ parasites are highlighted. Abbreviations: $\mathrm{BCKDH}$ : branched-chain a-keto acid dehydrogenase-complex.

Additional file 12 : Table S7. $\triangle B C K D H$ proteome. Excel spreadheet summarising the proteome data set from the nano-LC-MS/MS analysis. Proteins which are significantly up- or downregulated in $\triangle B C K D H$ parasites are highlighted. Abbreviations: BCKDH: branched-chain a-keto acid dehydrogenase-complex; LC-MS/MS: liquid chromatography-tandem mass spectrometry.

Additional file 13 : Figure S6. Generation of parasites lacking BCKDH, FNT-1 or both. PDF image displaying the (a) schematic representation of the strategy to deplete the fnt- 1 locus and replace it with a dhfr-ts resistance cassette. (b) PCRs were performed on genomic DNA extracted from clones and using primers listed in Additional file 14: Table S8, confirming correct integration of the constructs. (c) Table summarising the growth defect in the various strains described throughout the manuscript. Abbreviations: BCKDH: branched-chain a-keto acid dehydrogenase-complex; FS: flanking sequence; DHFR-TS: dihydrofolate reductase-thymidylate synthase; FNT: formate/nitrite transporter; PCR: polymerase chain reaction; DNA: deoxyribonucleic acid; ACL: ATP-citrate lyase; ACS: acetyl-CoA synthetase; BCKDH: branched-chain a-keto acid dehydrogenase-complex; PEPCK: phosphoenolpyruvate carboxykinase-1.

Additional file 14 : Table S8. Primers. Excel spreadsheet providing an overview of the primers used in this publication including their sequences.

\section{Acknowledgements}

We would like to thank Fabien Sindikubwabo, Matteo Lunghi and Aarti Krishnan for the skilful technical assistance.

\section{Authors' contributions}

$J K, R D O$ and DS-F designed the experiments. JK, RDO, GS, P-JDB and SKD performed the experiments. JK, RDO, DJC, GS, YC, P-JDB and SKD analysed the data. JK and RDO wrote the original draft. JK, RDO, DS-F, DJC, YC, GS and SKD reviewed and edited the draft. DS-F, DJC, M-AH and YC supervised this work. DS-F, DJC, M-AH and YC acquired funding. All authors read and approved the final manuscript.

\section{Funding}

This research was supported by the European Research Council (ERC) under the European Union's Horizon 2020 research and innovation programme under Grant agreement no. 695596. Proteomic experiments were partly supported by the Agence Nationale de la Recherche (ANR-10-INBS-08-01 ProFI grant and ANR-12-BSV3-0009 TOXOHDAC grant). MAH was supported by the LabEx ParaFrap [ANR-11-LABX-0024] and the ERC [Consolidator Grant no. 614880 Hosting TOXO]. RDO was supported by the iGE3 program from the University of Geneva. The project was also supported by the SNSF (CRSIII_160702 and 51PHPO_157303). 


\section{Availability of data and materials}

All relevant data are enclosed in the manuscript and/or deposited online as outlined here. Raw and processed proteomic MS data have been deposited with the ProteomeXchange Consortium via the PRIDE partner repository, accession number PXD016133 [28]. Raw sequence read transcriptomic have been deposited in the European Nucleotide Archive, accession number PRJEB36162 [48]. Metabolomics MS data and search results have been deposited with the Metabolomics Workbench Consortium, accession number/Project ID: PR000885 [24].

\section{Ethics approval and consent to participate}

Not applicable.

\section{Competing interests}

The authors declare that they have no competing interests.

\section{Author details}

'Department of Microbiology and Molecular Medicine, CMU, University of Geneva, Rue Michel-Servet 1, 1211 Geneva, Switzerland. 'Drug Delivery, Disposition and Dynamics, Monash Institute of Pharmaceutical Sciences, Monash University, Parkville campus, Parkville, VIC 3052, Australia. ${ }^{3}$ University Grenoble Alpes, CEA, INSERM, IRIG, BGE, F-38000 Grenoble, France.

${ }^{4}$ Epigenetic and Parasites Team, UMR5163/LAPM, Domaine de la Merci, Jean Roget Institute, 38700 La Tronche, France.

\section{Received: 24 January 2020 Accepted: 8 May 2020}

Published online: 16 June 2020

\section{References}

1. Alday PH, Doggett JS. Drugs in development for toxoplasmosis: advances, challenges, and current status. Drug Des Devel Ther. 2017;11:273-93.

2. Blasco B, Leroy D, Fidock DA. Antimalarial drug resistance: linking Plasmodium falciparum parasite biology to the clinic. Nat Med. 2017;23(8): 917-28.

3. Ding M, Clayton C, Soldati D. Toxoplasma gondii catalase: are there peroxisomes in toxoplasma? J Cell Sci. 2000;113(Pt 13):2409-19.

4. Ludewig-Klingner AK, Michael V, Jarek M, Brinkmann H, Petersen J. Distribution and evolution of peroxisomes in alveolates (apicomplexa, dinoflagellates, ciliates). Genome Biol Evol. 2018;10(1):1-13.

5. Moog D, Przyborski JM, Maier UG. Genomic and proteomic evidence for the presence of a peroxisome in the apicomplexan parasite Toxoplasma gondii and other Coccidia. Genome Biol Evol. 2017;9(11):3108-21.

6. Choudhary C, Kumar C, Gnad F, Nielsen ML, Rehman M, Walther TC, et al. Lysine acetylation targets protein complexes and co-regulates major cellular functions. Science. 2009;325(5942):834-40.

7. Klemm SL, Shipony Z, Greenleaf WJ. Chromatin accessibility and the regulatory epigenome. Nat Rev Genet. 2019;20(4):207-20.

8. Narita T, Weinert BT, Choudhary C. Functions and mechanisms of nonhistone protein acetylation. Nat Rev Mol Cell Biol. 2019;20(3):156-74.

9. Pietrocola F, Galluzzi L, Bravo-San Pedro JM, Madeo F, Kroemer G. Acetyl coenzyme A: a central metabolite and second messenger. Cell Metab. 2015; 21(6):805-21.

10. Bushell E, Gomes AR, Sanderson T, Anar B, Girling G, Herd C, et al. Functional profiling of a Plasmodium genome reveals an abundance of essential genes. Cell. 2017;170(2):260-72 e8.

11. Sidik SM, Huet D, Ganesan SM, Huynh MH, Wang T, Nasamu AS, et al. A genome-wide CRISPR screen in toxoplasma identifies essential apicomplexan genes. Cell. 2016;166(6):1423-35 e12.

12. Fleige T, Fischer K, Ferguson DJ, Gross U, Bohne W. Carbohydrate metabolism in the Toxoplasma gondii apicoplast: localization of three glycolytic isoenzymes, the single pyruvate dehydrogenase complex, and a plastid phosphate translocator. Eukaryot Cell. 2007;6(6):984-96.

13. Oppenheim RD, Creek DJ, Macrae Jl, Modrzynska KK, Pino P, Limenitakis J, et al. BCKDH: the missing link in apicomplexan mitochondrial metabolism is required for full virulence of Toxoplasma gondii and Plasmodium berghei. PLoS Pathog. 2014;10(7):e1004263.

14. Tymoshenko S, Oppenheim RD, Agren R, Nielsen J, Soldati-Favre D, Hatzimanikatis V. Metabolic needs and capabilities of toxoplasma gondii through combined computational and experimental analysis. PLoS Comput Biol. 2015;11(5):e1004261.
15. Kanamori A, Nakayama J, Fukuda MN, Stallcup WB, Sasaki K, Fukuda M, et al. Expression cloning and characterization of a CDNA encoding a novel membrane protein required for the formation of O-acetylated ganglioside: a putative acetyl-CoA transporter. Proc Natl Acad Sci U S A. 1997:94(7):2897-902.

16. Krishnan A, Kloehn J, Lunghi M, Chiappino-Pepe A, Waldman BS, Nicolas D, et al. Functional and computational genomics reveal unprecedented flexibility in stage-specific Toxoplasma metabolism. Cell Host Microbe. 2020; 27(2):290-306 e11.

17. Schalkwijk J, Allman EL, Jansen PAM, de Vries LE, Verhoef JMJ, Jackowski S, et al. Antimalarial pantothenamide metabolites target acetyl-coenzyme A biosynthesis in Plasmodium falciparum. Sci Transl Med. 2019;11(510): eaas9917.

18. Zhang M, Wang C, Otto TD, Oberstaller J, Liao X, Adapa SR, et al. Uncovering the essential genes of the human malaria parasite Plasmodium falciparum by saturation mutagenesis. Science. 2018;360(6388):eaap7847.

19. Cobbold SA, Santos JM, Ochoa A, Perlman DH, Llinas M. Proteome-wide analysis reveals widespread lysine acetylation of major protein complexes in the malaria parasite. Sci Rep. 2016;6:19722.

20. Jeffers $V$, Sullivan WJ Jr. Lysine acetylation is widespread on proteins of diverse function and localization in the protozoan parasite Toxoplasma gondii. Eukaryot Cell. 2012;11(6):735-42.

21. Wang ZX, Hu RS, Zhou CX, He JJ, Elsheikha HM, Zhu XQ. Label-free quantitative acetylome analysis reveals Toxoplasma gondii genotypespecific acetylomic signatures. Microorganisms. 2019;7:510.

22. Xue B, Jeffers V, Sullivan WJ, Uversky VN. Protein intrinsic disorder in the acetylome of intracellular and extracellular Toxoplasma gondii. Mol BioSyst. 2013:9(4):645-57.

23. Herm-Gotz A, Agop-Nersesian C, Munter S, Grimley JS, Wandless TJ, Frischknecht F, et al. Rapid control of protein level in the apicomplexan toxoplasma gondii. Nat Methods. 2007:4(12):1003-5.

24. Siddiqui G. Multi-omics analysis delineates the distinct functions of sub-cellular acetyl-CoA pools in Toxoplasma gondii. Metabolomics Workbench Consortium 2020. https:/www.metabolomicsworkbench.org/data/DRCCMetadata. php?Mode=Project\&ProjectID=PR000885. Accessed 20 May 2020.

25. Ramakrishnan S, Docampo MD, MacRae JI, Ralton JE, Rupasinghe T, McConville MJ, et al. The intracellular parasite Toxoplasma gondii depends on the synthesis of long-chain and very long-chain unsaturated fatty acids not supplied by the host cell. Mol Microbiol. 2015;97(1):64-76.

26. Pino P, Foth BJ, Kwok LY, Sheiner $L$, Schepers $R$, Soldati $T$, et al. Dual targeting of antioxidant and metabolic enzymes to the mitochondrion and the apicoplast of Toxoplasma gondii. PLoS Pathog. 2007;3(8):e115.

27. Barylyuk K, Koreny L, Ke H, Butterworth S, Crook OM, Lassadi I, et al. A subcellular atlas of Toxoplasma reveals the functional context of the proteome. Preprint at bioRxiv. 2020. https://doi.org/10.1101/2020.04.23. 057125 .

28. Coute Y. Elucidating the diverse roles of acetyl-CoA in the cytosol and mitochondrion of Toxoplasma gondii. ProteomeXchange Consortium via the PRIDE partner Repository 2020. http://proteomecentral. proteomexchange.org/cgi/GetDataset?ID=PXD016133. Accessed 20 May 2020.

29. Alexa A, Rahnenfuhrer J. Gene set enrichment analysis with topGO; 2007.

30. Naguleswaran A, Elias EV, McClintick J, Edenberg HJ, Sullivan WJ Jr. Toxoplasma gondii lysine acetyltransferase GCN5-A functions in the cellular response to alkaline stress and expression of cyst genes. PLoS Pathog. 2010; 6(12):e1001232.

31. Smith AT, Tucker-Samaras SD, Fairlamb AH, Sullivan WJ Jr. MYST family histone acetyltransferases in the protozoan parasite Toxoplasma gondii. Eukaryot Cell. 2005;4(12):2057-65.

32. Vanagas $L$, Jeffers $V$, Bogado SS, Dalmasso MC, Sullivan WJ Jr, Angel SO. Toxoplasma histone acetylation remodelers as novel drug targets. Expert Rev Anti-Infect Ther. 2012;10(10):1189-201.

33. Wang J, Dixon SE, Ting LM, Liu TK, Jeffers V, Croken MM, et al. Lysine acetyltransferase GCN5b interacts with AP2 factors and is required for Toxoplasma gondii proliferation. PLoS Pathog. 2014;10(1):e1003830.

34. Jeninga MD, Quinn JE, Petter M. ApiAP2 transcription factors in apicomplexan parasites. Pathogens. 2019;8:47.

35. Farhat DC, Swale C, Dard C, Cannella D, Ortet P, Barakat M, et al. A MORCdriven transcriptional switch controls Toxoplasma developmental trajectories and sexual commitment. Nat Microbiol. 2020;5(4):570-83.

36. Kemp LE, Yamamoto M, Soldati-Favre D. Subversion of host cellular functions by the apicomplexan parasites. FEMS Microbiol Rev. 2013;37(4):607-31. 
37. Finkemeier I, Laxa M, Miguet L, Howden AJ, Sweetlove LJ. Proteins of diverse function and subcellular location are lysine acetylated in Arabidopsis. Plant Physiol. 2011;155(4):1779-90.

38. Wang $Q$, Zhang Y, Yang C, Xiong H, Lin Y, Yao J, et al. Acetylation of metabolic enzymes coordinates carbon source utilization and metabolic flux. Science. 2010;327(5968):1004-7.

39. Zhao $S, X u$ W, Jiang W, Yu W, Lin Y, Zhang T, et al. Regulation of cellular metabolism by protein lysine acetylation. Science. 2010;327(5968):1000-4.

40. Cha Y, Han MJ, Cha HJ, Zoldan J, Burkart A, Jung JH, et al. Metabolic control of primed human pluripotent stem cell fate and function by the miR-200cSIRT2 axis. Nat Cell Biol. 2017;19(5):445-56.

41. Nakayasu ES, Burnet MC, Walukiewicz HE, Wilkins CS, Shukla AK, Brooks S, et al. Ancient regulatory role of lysine acetylation in central metabolism. MBio. 2017;8(6):e01894-17.

42. Nitzsche R, Gunay-Esiyok O, Tischer M, Zagoriy V, Gupta N. A plant/fungaltype phosphoenolpyruvate carboxykinase located in the parasite mitochondrion ensures glucose-independent survival of Toxoplasma gondii. J Biol Chem. 2017;292(37):15225-39.

43. Guan $\mathrm{KL}$, Xiong Y. Regulation of intermediary metabolism by protein acetylation. Trends Biochem Sci. 2011;36(2):108-16.

44. Zhao D, Zou SW, Liu Y, Zhou X, Mo Y, Wang P, et al. Lysine-5 acetylation negatively regulates lactate dehydrogenase $A$ and is decreased in pancreatic cancer. Cancer Cell. 2013;23(4):464-76.

45. Blume M, Nitzsche R, Sternberg U, Gerlic M, Masters SL, Gupta N, et al. A Toxoplasma gondii gluconeogenic enzyme contributes to robust central carbon metabolism and is essential for replication and virulence. Cell Host Microbe. 2015;18(2):210-20

46. MacRae Jl, Sheiner L, Nahid A, Tonkin C, Striepen B, McConville MJ. Mitochondrial metabolism of glucose and glutamine is required for intracellular growth of Toxoplasma gondii. Cell Host Microbe. 2012;12(5): 682-92

47. Nitzsche R, Zagoriy V, Lucius R, Gupta N. Metabolic cooperation of glucose and glutamine is essential for the lytic cycle of obligate intracellular parasite Toxoplasma gondii. J Biol Chem. 2016;291(1):126-41.

48. Dogga SK. Toxoplasma gondii ACL and ACS - contribution to subcellular acetyl-CoA pools. European Nucleotide Archive. 2020; https://www.ebi.ac. uk/ena/data/view/PRJEB36162. Accessed 20 May 2020.

49. Martins-Duarte ES, Carias M, Vommaro R, Surolia N, de Souza W. Apicoplast fatty acid synthesis is essential for pellicle formation at the end of cytokinesis in Toxoplasma gondii. J Cell Sci. 2016;129(17):3320-31.

50. Frenal K, Jacot D, Hammoudi PM, Graindorge A, Maco B, Soldati-Favre D. Myosin-dependent cell-cell communication controls synchronicity of division in acute and chronic stages of Toxoplasma gondii. Nat Commun. 2017:8:15710.

51. Erler H, Ren B, Gupta N, Beitz E. The intracellular parasite Toxoplasma gondii harbors three druggable FNT-type formate and I-lactate transporters in the plasma membrane. J Biol Chem. 2018;293(45):17622-30.

52. Golldack A, Henke B, Bergmann B, Wiechert M, Erler H, Blancke Soares A, et al. Substrate-analogous inhibitors exert antimalarial action by targeting the Plasmodium lactate transporter PfFNT at nanomolar scale. PLoS Pathog. 2017;13(2):e1006172.

53. Hapuarachchi SV, Cobbold SA, Shafik SH, Dennis AS, McConville MJ, Martin $\mathrm{RE}$, et al. The malaria parasite's lactate transporter PfFNT is the target of antiplasmodial compounds identified in whole cell phenotypic screens. PLoS Pathog. 2017;13(2):e1006180.

54. Marchetti RV, Lehane AM, Shafik SH, Winterberg M, Martin RE, Kirk K. A lactate and formate transporter in the intraerythrocytic malaria parasite, Plasmodium falciparum. Nat Commun. 2015;6:6721.

55. Wu B, Rambow J, Bock S, Holm-Bertelsen J, Wiechert M, Soares AB, et al. Identity of a Plasmodium lactate/ $\mathrm{H}(+)$ symporter structurally unrelated to human transporters. Nat Commun. 2015;6:6284.

56. Trenholme K, Marek L, Duffy S, Pradel G, Fisher G, Hansen FK, et al. Lysine acetylation in sexual stage malaria parasites is a target for antimalarial small molecules. Antimicrob Agents Chemother. 2014:58(7):3666-78.

57. Dubois D, Fernandes S, Amiar S, Dass S, Katris NJ, Botte CY, et al. Toxoplasma gondii acetyl-CoA synthetase is involved in fatty acid elongation (of long fatty acid chains) during tachyzoite life stages. J Lipid Res. 2018;59(6):994-1004.

58. Fox BA, Ristuccia JG, Gigley JP, Bzik DJ. Efficient gene replacements in toxoplasma gondii strains deficient for nonhomologous end joining. Eukaryot Cell. 2009;8(4):520-9.
59. Shen B, Brown KM, Lee TD, Sibley LD. Efficient gene disruption in diverse strains of Toxoplasma gondii using CRISPR/CAS9. MBio. 2014; 5(3):e01114-14

60. Santos JM, Ferguson DJ, Blackman MJ, Soldati-Favre D. Intramembrane cleavage of AMA1 triggers Toxoplasma to switch from an invasive to a replicative mode. Science. 2011;331(6016):473-7.

61. Sheiner L, Santos JM, Klages N, Parussini F, Jemmely N, Friedrich N, et al. Toxoplasma gondii transmembrane microneme proteins and their modular design. Mol Microbiol. 2010;77(4):912-29.

62. Bullen HE, Jia Y, Yamaryo-Botte $Y$, Bisio H, Zhang O, Jemelin NK, et al. Phosphatidic acid-mediated signaling regulates microneme secretion in Toxoplasma. Cell Host Microbe. 2016;19(3):349-60.

63. Soldati D, Boothroyd JC. Transient transfection and expression in the obligate intracellular parasite Toxoplasma gondii. Science. 1993;260(5106): 349-52.

64. Frenal K, Marq JB, Jacot D, Polonais V, Soldati-Favre D. Plasticity between MyoC- and MyoA-glideosomes: an example of functional compensation in Toxoplasma gondii invasion. PLoS Pathog. 2014;10(10): e1004504.

65. Plattner F, Yarovinsky F, Romero S, Didry D, Carlier MF, Sher A, et al. Toxoplasma profilin is essential for host cell invasion and TLR11dependent induction of an interleukin-12 response. Cell Host Microbe. 2008;3(2):77-87.

66. Herm-Gotz A, Weiss S, Stratmann R, Fujita-Becker S, Ruff C, Meyhofer E, et al. Toxoplasma gondii myosin A and its light chain: a fast, single-headed, plusend-directed motor. EMBO J. 2002;21(9):2149-58.

67. Bastin P, Bagherzadeh Z, Matthews KR, Gull K. A novel epitope tag system to study protein targeting and organelle biogenesis in Trypanosoma brucei. Mol Biochem Parasitol. 1996;77(2):235-9.

68. Jacot D, Daher W, Soldati-Favre D. Toxoplasma gondii myosin F, an essential motor for centrosomes positioning and apicoplast inheritance. EMBO J. 2013:32(12):1702-16.

69. DeRocher AE, Coppens I, Karnataki A, Gilbert LA, Rome ME, Feagin JE, et al. A thioredoxin family protein of the apicoplast periphery identifies abundant candidate transport vesicles in Toxoplasma gondii. Eukaryot Cell. 2008;7(9): 1518-29.

70. Dogga SK, Mukherjee B, Jacot D, Kockmann T, Molino L, Hammoudi PM, et al. A druggable secretory protein maturase of Toxoplasma essential for invasion and egress. Elife. 2017;6:e27480.

71. Anders S, Pyl PT, Huber W. HTSeq--a Python framework to work with highthroughput sequencing data. Bioinformatics. 2015;31(2):166-9.

72. Kim D, Pertea G, Trapnell C, Pimentel H, Kelley R, Salzberg SL. TopHat2: accurate alignment of transcriptomes in the presence of insertions, deletions and gene fusions. Genome Biol. 2013;14(4):R36.

73. Langmead B, Salzberg SL. Fast gapped-read alignment with Bowtie 2. Nat Methods. 2012;9(4):357-9.

74. Trapnell C, Pachter L, Salzberg SL. TopHat: discovering splice junctions with RNA-Seq. Bioinformatics. 2009;25(9):1105-11.

75. Perez-Riverol Y, Csordas A, Bai J, Bernal-Llinares M, Hewapathirana S, Kundu DJ, et al. The PRIDE database and related tools and resources in 2019: improving support for quantification data. Nucleic Acids Res. 2019;47(D1): D442-D50,

76. Tyanova S, Temu T, Cox J. The MaxQuant computational platform for mass spectrometry-based shotgun proteomics. Nat Protoc. 2016;11(12):2301-19.

77. Gajria B, Bahl A, Brestelli J, Dommer J, Fischer S, Gao X, et al. ToxoDB: an integrated Toxoplasma gondii database resource. Nucleic Acids Res. 2008; 36(Database issue):D553-6.

78. Wieczorek S, Combes F, Lazar C, Giai Gianetto Q, Gatto L, Dorffer A, et al. DAPAR \& ProStaR: software to perform statistical analyses in quantitative discovery proteomics. Bioinformatics. 2017;33(1):135-6.

79. Creek DJ, Chua HH, Cobbold SA, Nijagal B, MacRae Jl, Dickerman BK, et al. Metabolomics-based screening of the malaria box reveals both novel and established mechanisms of action. Antimicrob Agents Chemother. 2016; 60(11):6650-63.

80. Creek DJ, Jankevics A, Burgess KE, Breitling R, Barrett MP. IDEOM: an Excel interface for analysis of LC-MS-based metabolomics data. Bioinformatics. 2012;28(7):1048-9.

81. Creek DJ, Jankevics A, Breitling R, Watson DG, Barrett MP, Burgess KE. Toward global metabolomics analysis with hydrophilic interaction liquid chromatography-mass spectrometry: improved metabolite identification by retention time prediction. Anal Chem. 2011;83(22):8703-10. 
82. Chokkathukalam A, Jankevics A, Creek DJ, Achcar F, Barrett MP, Breitling R. mzMatch-ISO: an $\mathrm{R}$ tool for the annotation and relative quantification of isotope-labelled mass spectrometry data. Bioinformatics. 2013;29(2):281-3.

83. Zamboni N, Fendt SM, Ruhl M, Sauer U. (13)C-based metabolic flux analysis. Nat Protoc. 2009;4(6):878-92.

\section{Publisher's Note}

Springer Nature remains neutral with regard to jurisdictional claims in published maps and institutional affiliations.

Ready to submit your research? Choose BMC and benefit from:

- fast, convenient online submission

- thorough peer review by experienced researchers in your field

- rapid publication on acceptance

- support for research data, including large and complex data types

- gold Open Access which fosters wider collaboration and increased citations

- maximum visibility for your research: over $100 \mathrm{M}$ website views per year

At BMC, research is always in progress.

Learn more biomedcentral.com/submissions 